RESEARCH ARTICLE

\author{
D. D. Anderson • R. M. Ortiz-Albino
}

\title{
Three frameworks for a general theory of factorization
}

Received: 14 November 2010 / Accepted: 9 June 2011 / Published online: 24 March 2012

(C) The Author(s) 2012. This article is published with open access at Springerlink.com

\begin{abstract}
We discuss three different frameworks for a general theory of factorization in integral domains: $\tau$-factorization, reduced $\tau$-factorization and $\Gamma$-factorization. Let $D$ be an integral domain, $D^{\sharp}$ the non-zero, non-units of $D$, and $\tau$ a symmetric relation on $D^{\sharp}$. For $a \in D^{\sharp}, a=\lambda a_{1} \cdots a_{n}, \lambda$ a unit, $a_{i} \in D^{\sharp}, n \geq 1$, and $a_{i} \tau a_{j}$ for $i \neq j$, is called a $\tau$-factorization of $a$ and we say $a_{i}$ is a $\tau$-factor of $a$. For $a, b \in D^{\sharp},\left.a\right|_{\tau} b$ if $a$ is a $\tau$-factor of $b$. Then $a \in D^{\sharp}$ is a $\tau$-atom if any $\tau$-factorization of $a$ has $n=1$ and $a$ is a $\tau$-prime (resp., $\left.\right|_{\tau}$-prime) if $a \mid \lambda a_{1} \cdots a_{n}$ (resp., $\left.a\right|_{\tau} \lambda a_{1} \cdots a_{n}$ ), $\lambda a_{1} \cdots a_{n}$ a $\tau$-factorization, implies $a \mid a_{i}$ (resp., $\left.a\right|_{\tau} a_{i}$ ) for some $i$. The theory of reduced $\tau$-factorization is developed similarly, except here we restrict ourselves to reduced $\tau$-factorizations, that is, $\tau$-factorizations $a_{1} \cdots a_{n}$ where the leading unit is omitted (or is 1 ). The theory of $\Gamma$-factorization is as follows. For $a \in D^{\sharp}$, fact $(a)$ (resp., tfact $(a)$ ) is the set of (resp., trivial) factorizations of $a, a=\lambda a_{1} \cdots a_{n}, \lambda$ a unit, $n \geq 1$ (resp,. $\left.n=1\right)$ and fact $(D)=\cup_{a \in D^{\sharp}} \operatorname{fact}(a), \operatorname{tfact}(D)=\cup_{a \in D^{\sharp}} \operatorname{tfact}(a)$. Let $\Gamma \subseteq \operatorname{fact}(D)$ and $\Gamma(a)=\Gamma \cap \operatorname{fact}(a)$; the set of $\Gamma$-factorizations of $a$. For $a, b \in D^{\sharp},\left.a\right|_{\Gamma} b$ if some $\lambda a_{1} \cdots a_{n} \in \Gamma(b)$ has $a_{i}=a$ for some $i$. We say $a$ is a $\Gamma$-atom if $\Gamma(a) \subseteq \operatorname{tfact}(a)$ and that $a$ is a $\Gamma$-prime

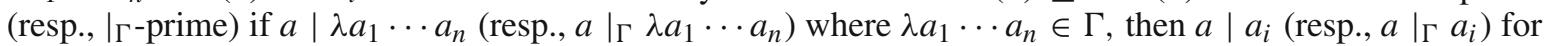
some $i$.
\end{abstract}

Mathematics Subject Classification (2010) 13A05 - 13G05 · 13F15

D. D. Anderson $(\varangle)$

Department of Mathematics, The University of Iowa,

Iowa City, IA 52242, USA

E-mail: dan-anderson@uiowa.edu

R. M. Ortiz-Albino

Department of Mathematics, University of Puerto Rico,

Mayaguez, 00681 Puerto Rico

E-mail: reyes.ortiz@upr.edu 


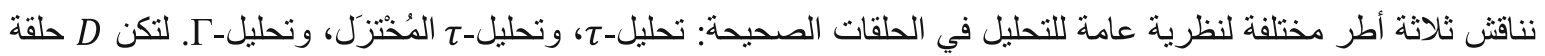

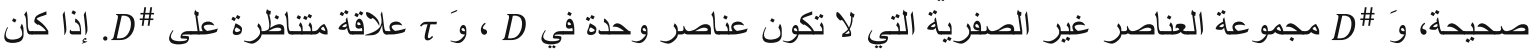

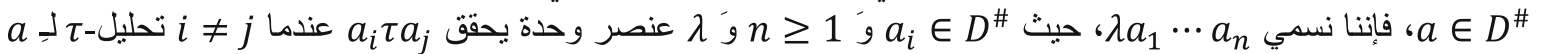
ونقول أن a $a_{i}$

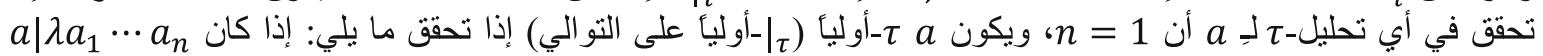

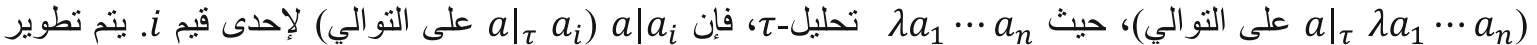

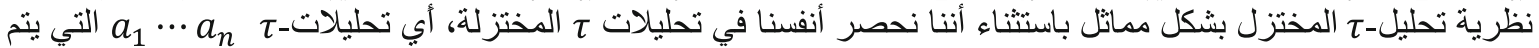

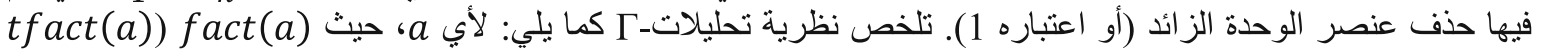

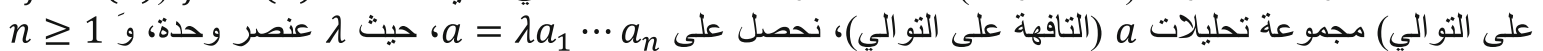

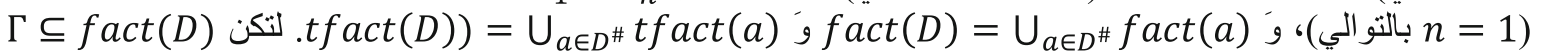

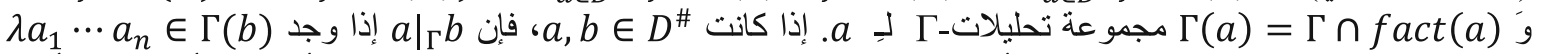
بحيث يكون $a$ act(a) على التوالي) في حال تحقق ما يلي: إذا كان

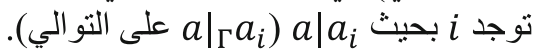

\section{Introduction}

The purpose of this paper is to give three different approaches to a general theory of factorization in integral domains. We isolate four important notions in factorization: a factorization, the related notion of divides, an atom or irreducible element, and a principal prime element. Let $D$ be an integral domain, $U(D)$ the group of units of $D$, and $D^{\sharp}=D-(\{0\} \cup U(D))$, the non-zero, non-units of $D$. For $a \in D^{\sharp}, a=a_{1} \cdots a_{n}$, or more generally, $a=\lambda a_{1} \cdots a_{n}, n \geq 1, \lambda \in U(D), a_{i} \in D^{\sharp}$, is a factorization of $a$ and we say $a_{i}$ is a factor of $a$. For $a, b \in D^{\sharp}, a \mid b$ if $a$ is a factor of $b$. Then $a \in D^{\sharp}$ is irreducible or an atom if for any factorization $a=\lambda a_{1} \cdots a_{n}$ of $a$, we have $n=1$. Finally, $a \in D^{\sharp}$ is prime if whenever $a \mid \lambda a_{1} \cdots a_{n}, \lambda a_{1} \cdots a_{n}$ a factorization, then $a \mid a_{i}$ for some $i$.

Now classically, factorization theory considered factorization into atoms or atomic factorization. An integral domain $D$ is atomic if each element of $D^{\sharp}$ is a product of atoms. One then studies atomic domains with properties weaker than unique factorization. For example, an atomic domain $D$ is a half-factorial domain (HFD) if any two atomic factorizations of $a \in D^{\sharp}$ have the same length and a (an atomic) domain $D$ is a bounded factorization domain (BFD) if for each $a \in D^{\sharp}$, there is a natural number $N(a)$ so that for any (atomic) factorization $a_{1} \cdots a_{n}$ of $a$, we have $n \leq N(a)$. See [2] and [5] for details. However, one can also study non-atomic factorization. Instead of studying factorization into atoms, we could study factorization into primary elements or other distinguished elements. See [1] for details.

Instead of just varying the $a_{i}$ s allowed in a factorization $\lambda a_{1} \cdots a_{n}$, we can restrict the factorizations allowed. McAdam and Swan [10] did this in their study of comaximal factorization. For $a \in D^{\sharp}$, a comaximal factorization $a=a_{1} \cdots a_{n}$ of $a$ is a factorization where for $i \neq j, a_{i}$ and $a_{j}$ are comaximal, that is, $\left(a_{i}, a_{j}\right)=D$. They defined $a \in D^{\sharp}$ to be pseudo-irreducible if $a$ has no non-trivial comaximal factorization $a=b c$ (or equivalently, any comaximal factorization $a=\lambda a_{1} \cdots a_{n}$ of $a$ has $n=1$ ). They also defined $a \in D^{\sharp}$ to be pseudo-prime if $a \mid b c$ where $(b, c)=D$, then $a \mid b$ or $a \mid c$ (equivalently, if $a \mid \lambda a_{1} \cdots a_{n}$, a comaximal factorization, then $a \mid a_{i}$ for some $i$ ). They then studied domains called comaximal factorization domains (CFDs) with the property that every $a \in D^{\sharp}$ has a comaximal factorization into pseudo-irreducibles and CFDs called unique comaximal factorization domains (UCFDs) in which comaximal factorization into pseudo-irreducibles is unique up to order and associates.

In [3], the first author and A. Frazier introduced a general theory of factorization involving the notion of a $\tau$-factorization. Let $D$ be an integral domain and $\tau$ a symmetric relation on $D^{\sharp}$. For $a \in D^{\sharp}$, a $\tau$-factorization of $a$ is a factorization $a=\lambda a_{1} \cdots a_{n}$ where $a_{i} \tau a_{j}$ for $i \neq j$. For $a, b \in D^{\sharp}$, we say that $a \tau$-divides $b$, denoted $\left.a\right|_{\tau} b$, if $b$ has a $\tau$-factorization $b=\lambda a_{1} \cdots a_{n}$ where some $a_{i}=a$. We say that $a \in D^{\sharp}$ is a $\tau$-atom or $\tau$-irreducible if every $\tau$-factorization of $a$ is trivial: $a=\lambda\left(\lambda^{-1} a\right)$. Finally, $a \in D^{\sharp}$ is $\tau$-prime (resp., $\left.\right|_{\tau}$-prime) if for any $\tau$-factorization $\lambda a_{1} \cdots a_{n}$ with $a \mid \lambda a_{1} \cdots a_{n}$ (resp., $\left.a\right|_{\tau} \lambda a_{1} \cdots a_{n}$ ), then $a \mid a_{i}$ (resp., $\left.a\right|_{\tau} a_{i}$ ) for 
some $i$. For example, if we take $\tau=D^{\sharp} \times D^{\sharp}$, we get the ordinary factorization into non-units, while if we define $a \tau b \Leftrightarrow(a, b)=D$, we get the comaximal factorization of McAdam and Swan. Section 2 reviews the theory of $\tau$-factorization.

In the theory of $\tau$-factorization, the leading unit $\lambda$ in a $\tau$-factorization $\lambda a_{1} \cdots a_{n}$ turns out to play an important role. However, we could have developed the theory of $\tau$-factorizations by taking a $\tau$-factorization of $a \in D^{\sharp}$ to be $a=a_{1} \cdots a_{n}$ where $a_{i} \tau a_{j}$ for $i \neq j$. We call such a $\tau$-factorization a reduced $\tau$-factorization or a ${ }_{r} \tau$-factorization. An element $a \in D^{\sharp}$ is a reduced $\tau$-atom or a ${ }_{r} \tau$-atom if $a$ has only the trivial ${ }_{r} \tau$-factorization $a=a$ and $a$ is a reduced $\tau$-prime or ${ }_{r} \tau$-prime if $a \mid a_{1} \cdots a_{n}$, a ${ }_{r} \tau$-factorization, then $a \mid a_{i}$ for some $i$. The notion of reduced $\tau$-divides is more subtle. For $a, b \in D^{\sharp}, a$ reduced $\tau$-divides $b$, denoted $\left.a\right|_{r \tau} b$, if $b=a_{1} \cdots a_{n}, a_{r} \tau$-factorization, where some $a_{i}=a$ and $a$ weakly reduced $\tau$-divides $b$, denoted $\left.a\right|_{w r} \tau b$, if $\left.a\right|_{r} \tau b$ or $a \sim b$. Finally, $a \in D^{\sharp}$ is a $\left.\right|_{r} \tau$-prime (resp., $\left.\right|_{w r} \tau$-prime) if $\left.a\right|_{r} \tau a_{1} \cdots a_{n}$ (resp., $\left.a\right|_{w r} \tau a_{1} \cdots a_{n}$ ), a ${ }_{r} \tau$-factorization, implies $\left.a\right|_{r} \tau a_{i}$ (resp., $\left.a\right|_{w r} \tau a_{i}$ ) for some $i$. The theory of reduced $\tau$-factorization is developed in Sect. 3. We believe that the results of Sect. 3 bear out that the choice of allowing a unit in the definition of a $\tau$-factorization is the proper one.

In the final Sect. 4, we introduce the notion of $\Gamma$-factorization. Let $D$ be an integral domain and $a \in D^{\sharp}$. By a (trivial) factorization of $a$, we mean $a=\lambda a_{1} \cdots a_{n}$ where $n \geq 1(n=1), \lambda \in U(D)$, and $a_{i} \in D^{\sharp}$ where the order matters (see Remark 2 of Sect. 4 for a more formal definition). Let fact $(a)$ (resp., $\operatorname{tfact}(a))$ be the set of all factorizations (resp., trivial factorizations) of $a$ and let fact $(D)=\cup_{a \in D^{\sharp}} \operatorname{fact}(a)$. Let $\Gamma \subseteq \operatorname{fact}(D)$. An element of $\Gamma$ (resp., $\Gamma(a):=\Gamma \cap$ fact $(a)$ ) is called a $\Gamma$-factorization (resp., $\Gamma$-factorization of $a$ ). For $a, b \in D^{\sharp}$, we say that $a \Gamma$-divides $b$, written $\left.a\right|_{\Gamma} b$, if there is a $\lambda a_{1} \cdots a_{n} \in \Gamma(b)$ with some $a_{i}=a$. Then, $a$ is a $\Gamma$-atom if $\Gamma(a) \subseteq \operatorname{tfact}(a)$ and $a$ is a $\Gamma$-prime (resp., $\left.\right|_{\Gamma}$-prime) if whenever $a \mid \lambda a_{1} \cdots a_{n}$ (resp., $\left.a\right|_{\Gamma} \lambda a_{1} \cdots a_{n}$ ), $\lambda a_{1} \cdots a_{n} \in \Gamma$, then $a \mid a_{i}$ (resp., $\left.a\right|_{\Gamma} a_{i}$ ) for some $i$. Suppose that $\tau$ is a symmetric relation on $D^{\sharp}$. If we take $\Gamma=\Gamma_{\tau}$ (resp., $\Gamma=\Gamma_{r} \tau$ ) to be the set of all $\tau$-factorizations (resp., reduced $\tau$-factorizations), we recover the notion of $\tau$-factorization (resp., reduced $\tau$-factorization).

\section{$2 \tau$-Factorizations}

In this section, we give a brief review of the theory of $\tau$-factorizations. Proofs and examples for claims may be found in Sect. 2 of [3] unless otherwise noted. Much of the material from [3] comes from [4]. The theory of $\tau$-factorizations is further investigated in the dissertations [7,9,11] and [12].

Throughout $D$ will be an integral domain with quotient field $K$. Let $D^{*}=D-\{0\}, U(D)$ the group of units of $D$, and $D^{\sharp}=D^{*}-U(D)$, the non-zero, non-units of $D$. Also throughout this section (except in the following definitions), $\tau$ will be a symmetric relation on $D^{\sharp}$. However, in Sect. 4 , we will consider ordered $\tau$-factorizations, the case where $\tau$ need not be symmetric. We next define three important properties that $\tau$ may have. As usual, $a \sim b$ means that $a$ and $b$ are associates.

Definition 2.1 Let $D$ be an integral domain and $\tau$ a relation on $D^{\sharp}$. We call $\tau$ multiplicative (resp., divisive) if for $a, b, c \in D^{\sharp}$ (resp., $a, a^{\prime}, b, b^{\prime} \in D^{\sharp}$ ), $a \tau b$ and $a \tau c$ imply $a \tau b c$ and $b \tau a$ and $c \tau a$ imply $b c \tau a$ (resp., $a \tau b, a^{\prime} \mid a$ and $b^{\prime} \mid b$ imply $\left.a^{\prime} \tau b^{\prime}\right)$. We say that $\tau$ is associate preserving if for $a, b, b^{\prime} \in D^{\sharp}$ with $b \sim b^{\prime}, a \tau b$ implies $a \tau b^{\prime}$ and $b \tau a$ implies $b^{\prime} \tau a$.

We next give the fundamental definition of a $\tau$-factorization.

Definition 2.2 For $a \in D^{\sharp}, D$ be an integral domain and $\tau$ a relation on $D^{\sharp}$, we define $a=\lambda a_{1} \cdots a_{n}, n \geq$ $1, \lambda \in U(D), a_{i} \in D^{\sharp}$, to be a $\tau$-factorization of $a$ if $a_{i} \tau a_{j}$ for each $i \neq j$. We say that $a$ is a $\tau$-product of the $a_{i}$ and that $a_{i}$ is a $\tau$-factor of $a$. For $a, b \in D^{\sharp}$, we say that $a \tau$-divides $b$, written $\left.a\right|_{\tau} b$, if $a$ is a $\tau$-factor of $b$. We call $a=\lambda\left(\lambda^{-1} a\right)$ a trivial $\tau$-factorization of $a$.

Note that if $a=\lambda a_{1} \cdots a_{n}$ is a $\tau$-factorization, then so is each rearrangement $a=\lambda a_{\sigma(1)} \cdots a_{\sigma(n)}, \sigma \in S_{n}$. Also, observe that for $a, b \in D^{\sharp}$, the following conditions are equivalent: (1) $a \tau b$, (2) $a b$ is a $\tau$-factorization, (3) $\lambda a b$ is a $\tau$-factorization for all $\lambda \in U(D)$, (4) there is a $\tau$-factorization $\lambda a_{1} \cdots a_{n}$ where some $a_{i}=a$ and $a_{j}=b$ for $i \neq j$.

We pause to give several examples.

Example 2.3 Throughout $D$ will be an integral domain. 
(1) $\tau=D^{\sharp} \times D^{\sharp}$. This gives the usual notions of a factorization and divides. Of course, $\tau$ is both multiplicative and divisive.

(2) $\tau=\varnothing$. Here $a \in D^{\sharp}$ has only the trivial $\tau$-factorization and $\left.a\right|_{\tau} b \Leftrightarrow a \sim b$. Vacuously, $\tau$ is both multiplicative and divisive.

(3) Let $S$ be a non-empty subset of $D^{\sharp}$ and take $\tau=S \times S$, so $a \tau b \Leftrightarrow a, b \in S$. Here $\tau$ is multiplicative (divisive) if and only if $S$ is multiplicatively closed (closed under non-unit factors). A non-trivial $\tau$-factorization is up to unit factors just a factorization into elements from $S$. Thus, if we take $S$ to be the set of atoms of $D$, we get the usual factorization of an element into irreducible factors. In this case, every element of $D^{\sharp}$ is an atom or has a non-trivial $\tau$-factorization if and only if $D$ is atomic. We could also take $S$ to be the set of prime elements, prime power elements, primary elements, or other distinguished elements such as rigid elements or $t$-pure elements. These last examples are examples of non-atomic factorizations. See [1] for details. We could also replace $S$ by a subset $S^{\prime}$ where for each $s \in S$ there exists exactly one $s^{\prime} \in S^{\prime}$ with $s^{\prime} \sim s$. For example, for $D=\mathbb{Z}$, take $S$ to be the set of prime elements and take $S^{\prime}=\{n \in \mathbb{N} \mid n$ is prime\}. Here, $\tau$ is not associate preserving. Examples of this type are one of the reasons we chose to include a unit factor in the definition of a $\tau$-factorization. Sometimes, it is of interest to replace $S \times S$ by $S \times S-\Delta=\{(s, t) \in S \times S \mid s \neq t\}$. For example, if $P=\left\{p_{\alpha}\right\}$ is a set of non-associate primes, take $S=\left\{p_{\alpha}^{k} \mid p_{\alpha} \in P, k \geq 1\right\}$. Then for $\tau=S \times S-\Delta$, a non-trivial $\tau$-factorization is just a product $\lambda p_{\alpha_{1}}^{k_{1}} \cdots p_{\alpha_{n}}^{k_{n}}$ where $p_{\alpha_{1}}, \ldots, p_{\alpha_{n}}$ are distinct elements of $P$ and each $k_{i} \geq 1$.

(4) Let $I$ be an ideal of $D$ and define $a \tau b \Leftrightarrow a-b \in I$. A special case is $D=\mathbb{Z}$ and $I=(n)$, so $a \tau_{n} b \Leftrightarrow a \equiv b \bmod n$. Here, $\tau_{n}$ is multiplicative or associate preserving only for $n=2$ and is never divisive. The relation $\tau_{n}$ is investigated in $[3,4,7]$ and [11].

(5) Let $\star$ be a star-operation on $D$ and define $a \tau_{\star} b \Leftrightarrow(a, b)^{\star}=D$, that is, $a$ and $b$ are $\star$-coprime or $\star$-comaximal. (Recall that a star operation $\star$ on $D$ is a closure operation on the set of non-zero fractional ideals of $D$ that satisfies $(x A)^{\star}=x A^{\star}$ and $D^{\star}=D$, see [6]). It is easily checked that $\tau_{\star}$ is both multiplicative and divisive. In the case where $\star=d$ (the $d$-operation $A \longrightarrow A_{d}=A$ ), we have the comaximal factorization of McAdam and Swan [10]. Also of interest is the case where $\star$ is the $v$-operation $\left(A \rightarrow A_{v}=\left(A^{-1}\right)^{-1}\right)$.

(6) Related to factorizations into $v$-coprime elements, we have factorizations into relatively prime elements. Define for $a, b \in D^{\sharp} a \tau_{[]} b \Leftrightarrow[a, b]=1$, that is, $a$ and $b$ have no common non-unit factor. While divisive, $\tau_{[]}$need not be multiplicative. The relation $\tau_{[]}$is investigated in [11] and [12].

The notion of a $\tau$-factorization on $D$ like that of a topology on a set is very general. But part of the power of the definition is its generality. Of course, in topology we usually have other axioms such as the separation axioms. The analogy for $\tau$-factorization is the conditions that $\tau$ is associated-preserving, divisive, or multiplicative. We have found the divisive condition to be the most useful and is powerful enough to obtain interesting results such as Theorem 2.12.

Given a factorization, we often want to further factor certain terms or want to combine terms. In general, neither action preserves $\tau$-factorizations. Our first proposition states that if $\tau$ is divisive (resp., multiplicative), then the refinement of a $\tau$-factorization obtained by $\tau$-factoring one or more terms (resp., combining terms in a $\tau$-factorization) again gives a $\tau$-factorization. This good behavior was the main reason for introducing the notions of multiplicative and divisive relations.

Proposition 2.4 Let $D$ be an integral domain and let $\tau$ be a relation on $D^{\sharp}$.

(1) Suppose that $\tau$ is divisive. Let $a, b, b^{\prime} \in D^{\sharp}$ where $b \sim b^{\prime}$. Then $a \tau b \Leftrightarrow a \tau b^{\prime}$. So $\tau$ is associate preserving. Thus $a=\lambda a_{1} \cdots a_{n}$ is a $\tau$-factorization of $a$ if and only if $a_{1} \cdots\left(\lambda a_{i}\right) \cdots a_{n}$ is a $\tau$-factorization of $a$. Hence, when $\tau$ is divisive, or more generally associate preserving, we can dispense with the unit $\lambda$.

(2) Suppose that $\tau$ is divisive. Let $a=a_{1} \cdots a_{n}$ be a $\tau$-factorization of a and let $a_{i}=b_{i 1} \cdots b_{i m_{i}}$ be a $\tau$-factorization of $a_{i}$ (possibly the trivial factorization $a_{i}=b_{i 1}$ ). Then $a=b_{11} \cdots b_{1 m_{1}} b_{21} \cdots b_{2 m_{2}} \cdots b_{n 1} \cdots b_{n m_{n}}$ is a $\tau$-factorization of a, called a $\tau$-refinement of $a$. Thus when $\tau$ is divisive, a $\tau$-refinement of a $\tau$-factorization is a $\tau$-factorization.

(3) Suppose that $\tau$ is multiplicative. If $\{1,2, \ldots, n\}=A_{1} \dot{\cup} \ldots \dot{\cup} A_{s}$ (disjoint union) with each $A_{i}$ non-empty and $b_{i}=\Pi\left\{a_{j} \mid j \in A_{i}\right\}$, then $a=\lambda b_{1} \cdots b_{s}$ is a $\tau$-factorization of $a$.

We next discuss the relation $\left.\right|_{\tau}$ in more detail. Let $D$ be an integral domain and $\tau$ a relation on $D^{\sharp}$. Let $a, a^{\prime}, b, b^{\prime}, c \in D^{\sharp}$. Certainly, $\left.a\right|_{\tau} b \Rightarrow a \mid b$, but the converse is false. We have (1) $\left.a\right|_{\tau} a$ and (2) $\left.a\right|_{\tau} b$ and $\left.b\right|_{\tau} a \Leftrightarrow a \sim b$. If $b \sim b^{\prime}$, then $\left.\left.a\right|_{\tau} b \Leftrightarrow a\right|_{\tau} b^{\prime}$. If $a \sim a^{\prime}$ and $\tau$ is associate preserving, then $\left.\left.a\right|_{\tau} b \Leftrightarrow a^{\prime}\right|_{\tau} b$. However, in general $a \sim a^{\prime}$ and $\left.\left.a\right|_{\tau} b \nRightarrow a^{\prime}\right|_{\tau} b$. If $\tau$ is divisive, then (3) $\left.a\right|_{\tau} b$ and $\left.\left.b\right|_{\tau} c \Rightarrow a\right|_{\tau} c$. However, in general this is also false. If $\tau$ is both multiplicative and divisive, then $\left.a\right|_{\tau} b$ and $\left.b \tau c \Rightarrow a c\right|_{\tau} b c$. 
Definition 2.5 For $a \in D^{\sharp}, D$ an integral domain and $\tau$ a relation on $D^{\sharp}, a$ is $\tau$-irreducible or a $\tau$-atom if the only $\tau$-factorizations of $a$ are the trivial ones. Let $\tau^{\prime}$ be another relation on $D^{\sharp}$. By a $\tau$-atomic $\tau^{\prime}$-factorization for $a \in D^{\sharp}$, we mean a $\tau^{\prime}$-factorization of $a$ into $\tau$-atoms. We say that $D$ is $\tau$ - $\tau^{\prime}$ atomic if each $a \in D^{\sharp}$ has a $\tau$-atomic $\tau^{\prime}$-factorization. When $\tau=\tau^{\prime}$, we simply say a $\tau$-atomic factorization or that $D$ is $\tau$-atomic. We say that a $\tau$-factorization $a=\lambda a_{1} \cdots a_{n}$ is $\tau$-unrefineable or $\tau$-complete if it has no proper $\tau$-refinements. And $D$ is $\tau$-complete if every $a \in D^{\sharp}$ has a $\tau$-complete factorization.

Note that an associate of a $\tau$-atom is again a $\tau$-atom. Of course, a $\tau$-atomic factorization is $\tau$-complete. By Proposition 2.4(2) for $\tau$ divisive, a $\tau$-complete factorization is the same thing as a $\tau$-atomic factorization. This is not true in general. Hence for $\tau$ divisive, $D$ is $\tau$-complete if and only if it is $\tau$-atomic.

Definition 2.6 Let $\tau$ be a relation on $D^{\sharp}, D$ an integral domain. Then $a \in D^{\sharp}$ is $\tau$-prime if whenever $a \mid \lambda a_{1} \cdots a_{n}$ where $\lambda a_{1} \cdots a_{n}$ is a $\tau$-factorization, then $a \mid a_{i}$ for some $i$. We call $a \in D^{\sharp}$ a $\left.\right|_{\tau}$-prime if whenever $\left.a\right|_{\tau} \lambda a_{1} \cdots a_{n}$ where $\lambda a_{1} \cdots a_{n}$ is a $\tau$-factorization, then $\left.a\right|_{\tau} a_{i}$ for some $i$.

An associate of a $\tau$-prime element is again $\tau$-prime. If $\tau$ is associate preserving, then an associate of a $\left.\right|_{\tau}$-prime element is again $\left.\right|_{\tau}$-prime. However, in general an associate of a $\left.\right|_{\tau}$-prime element need not be $\left.\right|_{\tau}$-prime.

Remark 1 Note that $\tau$-primes and $\left.\right|_{\tau}$-primes are a special case of what we might call a $\tau_{1}-\tau_{2}$ - $\tau_{3}$-prime (where $\tau_{1}, \tau_{2}$ and $\tau_{3}$ are relations on $\left.D^{\sharp}\right)$ : whenever $\left.a\right|_{\tau_{2}} \lambda a_{1} \cdots a_{n}$ where $\lambda a_{1} \cdots a_{n}$ is a $\tau_{1}$-factorization, then $\left.a\right|_{\tau_{3}} a_{i}$ for some $i$. For example, a $\tau$-prime is a $\tau$ - $\tau^{\prime}$ - $\tau^{\prime}$-prime where $\tau^{\prime}=D^{\sharp} \times D^{\sharp}$ [Example 2.3(1)] and a $\left.\right|_{\tau}$-prime is a $\tau-\tau$ - $\tau$-prime.

Let $D$ be an integral domain, $\tau$ be a relation on $D^{\sharp}$, and $a \in D^{\sharp}$. Clearly if $a$ is irreducible (resp., prime), then $a$ is $\tau$-irreducible (resp., $\tau$-prime) and if $a$ is $\tau$-prime or $\left.\right|_{\tau}$-prime, then $a$ is $\tau$-irreducible. If $\tau$ is multiplicative and divisive, then $a \tau$-prime implies $a$ is $\left.\right|_{\tau}$-prime (see Proposition 2.7 below). But in general, a prime or $\tau$-prime element need not be $\left.\right|_{\tau}$-prime. Note that in the definitions of $\tau$-irreducible, $\tau$-prime, and $\left.\right|_{\tau}$-prime, we did not restrict ourselves to the case of $\tau$-factorizations $\lambda a_{1} \cdots a_{n}$ of length $n=2$ as is usual. We next note that if $\tau$ is multiplicative, we can restrict ourselves to the case $n=2$. In general this is not the case.

Proposition 2.7 Let $D$ be an integral domain and let $\tau$ be a relation on $D^{\sharp}$.

(1) Suppose that $\tau$ is multiplicative and let $a \in D^{\sharp}$. Then a is $\tau$-irreducible (resp., $\tau$-prime, $\left.\right|_{\tau}$-prime) if and only if a has no $\tau$-factorization $a=\lambda a_{1} a_{2}$ (resp., for a $\tau$-factorization $\lambda a_{1} a_{2}, a\left|\lambda a_{1} a_{2} \Rightarrow a\right| a_{1}$ or $\left.a\left|a_{2}, a\right|_{\tau} \lambda a_{1} a_{2} \Rightarrow a\right|_{\tau} a_{1}$ or $\left.\left.a\right|_{\tau} a_{2}\right)$.

(2) If $\tau$ is both multiplicative and divisive, then a $\tau$-prime element is $\left.\right|_{\tau}$-prime.

Let $D$ be an integral domain. For relations $\tau_{1}, \tau_{2}$ on $D^{\sharp}$, define $\tau_{1} \leq \tau_{2} \Leftrightarrow \tau_{1} \subseteq \tau_{2}$, that is, $a \tau_{1} b \Rightarrow a \tau_{2} b$. Observe that $\tau_{1} \leq \tau_{2}$ if and only if each $\tau_{1}$-factorization is a $\tau_{2}$-factorization. Let $\mathcal{R}$ be the set of relations on $D^{\sharp}$. So $\mathcal{R}$ is partially ordered by $\leq$. Note that $\varnothing$ [Example 2.3(2)] is the least element of $\mathcal{R}$ and the usual factorization is given by $\tau=D^{\sharp} \times D^{\sharp}$ [Example 2.3(1)], the greatest element. Suppose that $\star_{1}$ and $\star_{2}$ are two star-operations on $D$ with $\star_{1} \leq \star_{2}$, that is, $A^{\star_{1}} \subseteq A^{\star_{2}}$ for all $A \in F(D)$. Then $\tau_{\star_{1}} \leq \tau_{\star_{2}}$. Suppose that $\tau_{1}, \tau_{2}$ are relations on $D^{\sharp}$ with $\tau_{1} \leq \tau_{2}$. Then a $\tau_{1}$-factorization of $a \in D^{\sharp}$ is also a $\tau_{2}$-factorization of $a$. Thus if $a$ is a $\tau_{2}$-atom (resp., $\tau_{2}$-prime), then $a$ is a $\tau_{1}$-atom (resp., $\tau_{1}$-prime). Hence, we have the previously mentioned fact that an atom (resp., prime) of $D$ is a $\tau$-atom (resp., $\tau$-prime). Observe that if $\tau_{1} \leq \tau_{2}$, then $\left.\left.a\right|_{\tau_{1}} b \Rightarrow a\right|_{\tau_{2}} b$ for any $a, b \in D^{\sharp}$ and the converse is true if $\tau_{2}$ is both multiplicative and associate preserving, but not in general (define $\tau_{1}, \tau_{2}$ on $\mathbb{Z}^{\sharp}$ by $a \tau_{1} b \Leftrightarrow|a|=|b|=2$ and $2 \tau_{2} 2,2 \tau_{2}-2,-2 \tau_{2} 2$ ). However, a $\left.\right|_{\tau_{2}}$-prime need not be a $\left.\right|_{\tau_{1}}$-prime.

There is a natural extension of the notion of a UFD to $\tau$-factorizations. Let $D$ be an integral domain and $\tau$ a relation on $D^{\sharp}$. We say that $D$ is a $\tau$-UFD if (1) $D$ is $\tau$-atomic and (2) if $\lambda a_{1} \cdots a_{n}=\mu b_{1} \cdots b_{m}$ are two $\tau$-atomic factorizations, then $n=m$ and after re-ordering, if necessary, $a_{i} \sim b_{i}$ for each $i$. We leave it to the reader to define a $\tau$ - $\tau^{\prime}$-UFD using $\tau$-atomic $\tau^{\prime}$-factorizations. The following lemma used to prove Theorem 2.9 shows the importance of $\left.\right|_{\tau}$-primes.

Proposition 2.8 Let $D$ be an integral domain and $\tau$ a relation on $D^{\sharp}$. Suppose that $\lambda p_{1} \cdots p_{n}=\mu q_{1} \cdots q_{m}$ are two $\tau$-factorizations where the $p_{i}$ are $\left.\right|_{\tau}$-prime and the $q_{i}$ are $\tau$-atoms. Then $n=m$ and after re-ordering, if necessary, $p_{i} \sim q_{i}$. 
Theorem 2.9 Let $D$ be an integral domain and $\tau$ a relation on $D^{\sharp}$.

(1) Suppose that every element of $D^{\sharp}$ has a $\tau$-factorization into $\left.\right|_{\tau}$-primes. Then $D$ is a $\tau$-UFD. Moreover, $a \in D^{\sharp}$ is $\tau$-irreducible if and only if $a$ is an associate of $\left.a\right|_{\tau}$-prime.

(2) Suppose that $\tau$ is divisive and that $D$ is a $\tau$-UFD. Then a $\tau$-irreducible element of $D$ is $\left.\right|_{\tau}$-prime (and of course the converse always holds).

(3) For $\tau$ divisive, the following are equivalent:

(a) $D$ is a $\tau$-UFD,

(b) every element of $D^{\sharp}$ has a $\tau$-factorization into $\left.\right|_{\tau}$-primes, and

(c) $D$ is $\tau$-atomic and every $\tau$-irreducible element of $D$ is $\left.\right|_{\tau}$-prime.

In Theorem 2.9(3) if we replace $\left.\right|_{\tau}$-prime by $\tau$-prime, we have $(\mathrm{a}) \Leftarrow(\mathrm{b}) \Leftrightarrow(\mathrm{c})$, but $(\mathrm{a}) \nRightarrow(\mathrm{b})$. The counterexample for the implication (a) $\nRightarrow(b)$ is given in [9] which contains a thorough investigation of $\tau$-UFDs. However, a domain $D$ in which every element is a $\tau$-product of associates of $\left.\right|_{\tau}$-primes need not be a $\tau$-UFD. In a $\tau$-UFD every element of $D$ need not be a $\tau$-product of $\tau$-primes (resp., $\left.\right|_{\tau}$-primes).

We can also define the following $\tau$-factorization properties weaker than $\tau$-unique factorization. Let $D$ be an integral domain and $\tau$ a relation on $D^{\sharp}$. We have already defined $\tau$-atomic. We say that $D$ satisfies $\tau$-ACCP if for each infinite sequence $\left\{a_{n}\right\}_{n=1}^{\infty}$ of elements of $D^{\sharp}$ with $\left.a_{n+1}\right|_{\tau} a_{n}$ for each $n \geq 1$, there is an $N$ (depending on the sequence) with $a_{k+1} \sim a_{k}$ for each $k \geq N$. The domain $D$ is a $\tau$-half-factorial domain $(\tau-H F D)$ if $D$ is $\tau$-atomic and whenever $\lambda a_{1} \cdots a_{n}=\mu b_{1} \cdots b_{m}$ are two $\tau$-atomic factorizations, then $n=m$.

We say that $D$ is a $\tau$-bounded factorization domain $(\tau-B F D)$ if $D$ is $\tau$-atomic and for each $a \in D^{\sharp}$, there is a natural number $N(a)$ so that if $a=\lambda a_{1} \cdots a_{n}$ is a $\tau$-atomic factorization of $a$, then $n \leq N(a)$. Note that for $\tau$ divisive, $D$ is a $\tau$-BFD if and only if for each $a \in D^{\sharp}$, there is a natural number $N(a)$ so that for any $\tau$-factorization $a=\lambda a_{1} \cdots a_{n}, n \leq N(a)$. This follows from Proposition 2.4(2) which gives that for $\tau$ divisive a maximal length $\tau$-factorization is a $\tau$-atomic factorization. Thus for $\tau$ divisive, a BFD is a $\tau$-BFD.

We say that $D$ is a $\tau$-idf-domain if each $a \in D^{\sharp}$ has at most finitely many non-associate $\tau$-factors that are $\tau$-atoms. $D$ is a $\tau$-finite-factorization domain $(\tau-F F D)$ if $D$ is $\tau$-atomic and each $a \in D^{\sharp}$ has only finitely many $\tau$-factorizations (up to order and associates) into $\tau$-irreducibles. Clearly, a $\tau$-FFD is a $\tau$-BFD. Suppose that $\tau$ is divisive. Then a modification of the proof of [2, Theorem 5] gives that the following are equivalent: (1) $D$ is a $\tau$-FFD, (2) $D$ is a $\tau$-atomic $\tau$-idf-domain, (3) each $a \in D^{\sharp}$ has only finitely many $\tau$-factorizations up to order and associates.

Theorem 2.10 Let $D$ be an integral domain and $\tau$ a relation on $D^{\sharp}$. If $D$ is a $\tau-U F D$, then $D$ is a $\tau-H F D$ and a $\tau$-FFD and either of these conditions implies that $D$ is a $\tau-B F D$. If further $\tau$ is divisive, then $D$ a $\tau-B F D$ implies $D$ satisfies $\tau$-ACCP and if $D$ satisfies $\tau$-ACCP, then $D$ is $\tau$-atomic.

Let $D$ be an integral domain and $\tau$ a relation on $D^{\sharp}$. Note that if $D$ satisfies ACCP, then $D$ satisfies $\tau$-ACCP. Thus for $\tau$ divisive, ACCP $\Rightarrow \tau$-atomic. So for $\tau$ divisive, a UFD, FFD, HFD, and BFD are $\tau$-atomic. Thus a FFD (resp., BFD) is a $\tau$-FFD (resp., $\tau$-BFD) for $\tau$ divisive. However, an atomic domain need not be $\tau$-atomic, even if $\tau$ is both multiplicative and divisive. For [8] gives an example of an atomic domain that is not a CFD.

We next note that for $\tau$ divisive, a UFD is a $\tau$-UFD. Thus a UFD is a UCFD. The proof uses the following lemma which states that for $\tau$ divisive, a $\tau$-atomic factorization is a mix of atomic factorizations and coprime factorizations.

Lemma 2.11 Let $D$ be an integral domain and let $\tau$ be a divisive relation on $D^{\sharp}$. Let $a_{1} \cdots a_{n}$ be a $\tau$-atomic factorization. Then for $i \neq j$, either $\left[a_{i}, a_{j}\right]=1$ or $a_{i} \sim a_{j}$ are atoms.

Theorem 2.12 Let $D$ be a UFD and $\tau$ a divisive relation on $D^{\sharp}$. Then $D$ is a $\tau$-UFD.

As the following example shows, $D$ can be a $\tau$-UFD without $\tau$ being divisive. This example also shows that even in a $\tau$-UFD, the $\tau$ atoms do not determine $\left.\right|_{\tau}$.

Example 2.13 Let $D$ be a UFD and define $\tau$ on $D^{\sharp}$ by $a \tau b \Leftrightarrow a$ and $b$ are non-zero principal primes. So $\tau$ is divisive. Now clearly $D$ is a $\tau$-UFD with the sets of $\tau$-atoms, $\tau$-primes, $\left.\right|_{\tau}$-primes, and non-zero principal primes coinciding. Observe that $\left.a\right|_{\tau} b \Leftrightarrow a$ is a prime with $a \mid b$ or $a \sim b$. Suppose that $\tau \leq \tau^{\prime}$; so $\tau^{\prime}$ need not be divisive. Then $D$ is still a $\tau^{\prime}$-UFD with the set of $\tau^{\prime}$-atoms, $\tau^{\prime}$-primes, $\left.\right|_{\tau^{\prime}}$-primes, and non-zero principal primes coinciding. However, $\left.\right|_{\tau^{\prime}}$ may change. For if $p$ and $q$ are primes with $p / \tau^{\prime} \lambda q$ for any unit $\lambda$, then $\left.p\right|_{\tau} p q$, but $p \nmid_{\tau^{\prime}} p q$. 
We have based our factorization theory on $\tau$-atomic factorizations. We could have instead used $\tau$-complete factorizations. Let $D$ be an integral domain. Define $D$ to be a $\tau$-complete $H F D$ (resp., $\tau$-complete UFD) if (1) $D$ is $\tau$-complete and (2) if $a=\lambda a_{1} \cdots a_{n}=\mu b_{1} \cdots b_{m}$ are two $\tau$-complete factorizations of $a \in D^{\sharp}$, then $n=m$ (resp., and after re-ordering, if necessary, $a_{i} \sim b_{i}$ for $i=1, \ldots, n$ ). We say that $D$ is a $\tau$-complete FFD (resp., $\tau$-complete BFD) if for each $a \in D^{\sharp}$ there are only finitely many $\tau$-complete factorizations for $a$ up to units, order, and associates (resp., there is a natural number $N(a)$ so that for each $\tau$-complete factorization $\left.a=\lambda a_{1} \cdots a_{n}, n \leq N(a)\right)$.

Now even in a $\tau$-UFD, a $\tau$-factorization cannot necessarily be $\tau$-refined to a $\tau$-atomic factorization. Let us say that an integral domain $D$ is $\tau$-atomizable (resp., $\tau$-completeable) if each $\tau$-factorization of $D$ can be $\tau$-refined to a $\tau$-atomic (resp., $\tau$-complete) factorization. We have the following implications:

$$
\begin{aligned}
\tau \text {-atomizable } & \Rightarrow \tau \text {-atomic } \\
\Downarrow & \Downarrow \\
\tau \text {-ACCP } \Rightarrow \tau \text {-completeable } & \Rightarrow \tau \text {-complete. }
\end{aligned}
$$

Note that for $\tau$ divisive, $\tau$-complete $\Rightarrow \tau$-atomizable, but $\tau$-complete need not imply $\tau$-ACCP since an atomic domain need not satisfy ACCP. In general, none of the implications can be reversed. The topics in the previous two paragraphs are discussed in more detail in [9] in the context of $\Gamma$-factorization.

\section{Reduced $\tau$-factorization}

We maintain the notation from Sect. 2: $D$ is an integral domain and $\tau$ a symmetric relation on $D^{\sharp}$. In a $\tau$ factorization $a=\lambda a_{1} \cdots a_{n}$ for $a$ we allowed a leading unit $\lambda$. In this section we consider $\tau$-factorizations without the unit $\lambda$, or equivalently, where $\lambda=1$.

Definition 3.1 Let $D$ be an integral domain and $\tau, \tau_{1}$ and $\tau_{2}$ be relations on $D^{\sharp}$. For $a \in D^{\sharp}$, a reduced $\tau$-factorization (r $\tau$-factorization) of $a$ is $a=a_{1} \cdots a_{n}$ where $a_{i} \in D^{\sharp}$ and $a_{i} \tau a_{j}$ for $i \neq j$. The ${ }_{r} \tau$-factorization $a=a$ is called the trivial ${ }_{r} \tau$-factorization of $a$. So $a \in D^{\sharp}$ is a reduced $\tau$-atom or ${ }_{r} \tau$-atom if $a$ has only the trivial ${ }_{r} \tau$-factorization. A reduced $\tau_{1}$-atomic reduced $\tau_{2}$-factorization $\left(a_{r} \tau_{1}\right.$-atomic ${ }_{r} \tau_{2}$-factorization) of $a$ is a ${ }_{r} \tau_{2}$-factorization of $a$ into ${ }_{r} \tau_{1}$-atoms. Likewise, we define a ${ }_{r} \tau_{1}$-atomic $\tau_{2}$-factorization and $\tau_{1}$-atomic ${ }_{r} \tau_{2}$-factorization. A $\tau_{r} \tau_{1}$-atomic ${ }_{r} \tau_{1}$-factorization is a called $a_{r} \tau_{1}$-atomic factorization. We say that $D$ is ${ }_{r} \tau_{1-r} \tau_{2}$-atomic if each $a \in D^{\sharp}$ has a ${ }_{r} \tau_{1}$-atomic ${ }_{r} \tau_{2}$-factorization. Likewise, we define ${ }_{r} \tau_{1}-\tau_{2}$-atomic and $\tau_{1-r} \tau_{2}$-atomic. We say that $D$ is ${ }_{r} \tau$-atomic if $D$ is ${ }_{r} \tau-r \tau$-atomic.

The notion of a reduced $\tau$-factorization was introduced in [7] and investigated more fully in [11]. Much of this material comes from [11]. The following proposition states some simple facts about reduced $\tau$-factorizations and reduced $\tau$-atoms.

Proposition 3.2 Let $D$ be an integral domain and $\tau, \tau_{1}$ and $\tau_{2}$ be relations on $D^{\sharp}$.

(1) $A_{r} \tau$-factorization is a $\tau$-factorization.

(2) A $\tau$-atom is a ${ }_{r} \tau$-atom. So $D \tau_{1}-\tau_{2}$-atomic implies $D$ is ${ }_{r} \tau_{1}$ - $\tau_{2}$-atomic.

(3) Suppose that $\tau$ is associate preserving. Then $a_{r} \tau$-atom is a $\tau$-atom. So the following are equivalent: $(a)$ $D$ is $\tau$-atomic, (b) $D$ is $r_{r} \tau$ - $\tau$-atomic, (c) $D$ is $\tau$-r $\tau$-atomic, and (d) $D$ is ${ }_{r} \tau$-atomic.

(4) For $a \in D^{\sharp}$, a is a $\tau$-atom if and only if every associate of a is $a_{r} \tau$-atom.

Proof (1) Clear. (2) Suppose that $a$ is a $\tau$-atom. Then $a$ has only trivial $\tau$-factorizations and hence only the trivial $r \tau$-factorization. So $a$ is a $r \tau$-atom. The second statement is clear. (3) Let $a$ be a reduced $\tau$-atom. If $a$ is not a $\tau$-atom, we have a $\tau$-factorization $a=\lambda a_{1} \cdots a_{n}$ where $n>1$. But then $a=\left(\lambda a_{1}\right) \cdot a_{2} \cdots a_{n}$ is a non-trivial ${ }_{r} \tau$-factorization for $a$, a contradiction. So $a$ is a $\tau$-atom if and only if it is a $r \tau$-atom. For $a \in D^{\sharp}$, if $a$ has a $\tau$-atomic factorization $a=\lambda a_{1} \cdots a_{n}$, then $a=\left(\lambda a_{1}\right) \cdot a_{2} \cdots a_{n}$ is a $\tau$-atomic ${ }_{r} \tau$-factorization and hence a ${ }_{r} \tau$-atomic ${ }_{r} \tau$-factorization. The equivalence of (a)-(d) easily follows. (4) ( $\Rightarrow$ ) If $a$ is a $\tau$-atom, then each associate of $a$ is a $\tau$-atom and hence a ${ }_{r} \tau$-atom. $(\Leftarrow)$ Suppose that each associate of $a$ is a $r \tau$-atom. Suppose that is not a $\tau$-atom: so $a=\lambda a_{1} \cdots a_{n}$, a $\tau$-factorization with $n>1$. But then $\lambda^{-1} a=a_{1} \cdots a_{n}$ is a not a $r \tau$-atom; a contradiction.

We next give some examples. 
Example 3.3 Throughout, $D$ will be an integral domain.

(1) (An example of a $r \tau$-atom with an associate that is not a $r \tau$-atom and hence not a $\tau$-atom and a $\tau$-atomic domain that is not ${ }_{r} \tau$-atomic.) Define $\tau$ on $\mathbb{Z}^{\sharp}$ by $a \tau b \Leftrightarrow a, b \geq 2, a=4, b=-4$, or $a=-4, b=4$. Now each positive prime of $\mathbb{Z}$ is a $\tau$-atom; so $\mathbb{Z}$ is $\tau$-atomic. Here -4 is a $r \tau$-atom, but not a $\tau$-atom since $-4=(-1) \cdot 2 \cdot 2$ and the associate 4 of -4 is not a $r \tau$-atom. Now $-16=-4 \cdot 4$; so -16 is not a $r \tau$-atom. But there is no ${ }_{r} \tau$-atomic factorization for -16 (as $4=2 \cdot 2$ ). Thus $\mathbb{Z}$ is not ${ }_{r} \tau$-atomic and hence not $\tau-{ }_{r} \tau$-atomic.

(2) Define $\tau$ on $\mathbb{Z}^{\sharp}$ by $\pm p \tau \pm q$ where $p, q$ are any positive primes and $4 \tau 4$. So $\tau$ is not associate preserving. The $\tau$-atoms and $r \tau$-atoms are $\pm p$ where $p$ is a prime. Thus $\mathbb{Z}$ is $\tau$-atomic and ${ }_{r} \tau$-atomic and each associate of $\mathrm{a}_{r} \tau$-atom is a $r \tau$-atom; but $\tau$ is not associate preserving. Note that $\mathbb{Z}$ is a $\tau$-UFD and a ${ }_{r} \tau$-UFD (defined using $r \tau$-factorizations).

(3) Define $\tau$ on $\mathbb{Z}^{\sharp}$ by $p \tau q$ and $-p \tau-q$ where $p, q$ are any positive primes of $\mathbb{Z}$. The $\tau$-atoms are $\pm p, p$ a non-zero prime. Now $\mathbb{Z}$ is a $\tau$-UFD. The ${ }_{r} \tau$-atoms of $\mathbb{Z}$ are $\pm p$ and $-p_{1} \cdots p_{2 n}$ where $p$ and $p_{i}$ are positive primes. Note that $\mathbb{Z}$ is still a $r \tau$-UFD. If we add $-4 \tau-4$, then $-4 \cdot-4=2 \cdot 2 \cdot 2 \cdot 2$, so $\mathbb{Z}$ is no longer a $r \tau$-UFD, but is still a $\tau$-UFD.

Recall that $a \in D^{\sharp}$ is $\tau$-prime if whenever $a \mid \lambda a_{1} \cdots a_{n}$, a $\tau$-factorization, then $a \mid a_{i}$ for some $i$. We define $a \in D^{\sharp}$ to be ${ }_{r} \tau$-prime if $a \mid a_{1} \cdots a_{n}$, a ${ }_{r} \tau$-factorization, then $a \mid a_{i}$ for some $i$. Unlike the case for $\tau$-atoms and ${ }_{r} \tau$-atoms, the notions of $\tau$-prime and ${ }_{r} \tau$-prime coincide.

Proposition 3.4 Let $D$ be an integral domain and $\tau$ a symmetric relation on $D^{\sharp}$. Then $a \in D^{\sharp}$ is ${ }_{r} \tau$-prime if and only if it is $\tau$-prime. Hence $a_{r} \tau$-prime is $a_{r} \tau$-atom.

Proof $(\Leftarrow)$ This is immediate since a $r \tau$-factorization is a $\tau$-factorization. $(\Rightarrow)$ Suppose that $a$ is a ${ }_{r} \tau$-prime. Suppose that $a \mid \lambda a_{1} \cdots a_{n}$, a $\tau$-factorization. Then $a \mid a_{1} \cdots a_{n}$ where $a_{1} \cdots a_{n}$ is now a $r \tau$-factorization. So $a \mid a_{i}$ for some $i$. Thus $a$ is a $\tau$-prime.

We next want to consider "reduced $\left.\right|_{\tau}$-primes". To do this, we need to define "reduced $\tau$-divides". Now for $a, b \in D^{\sharp}$, the "natural" way to do this is to define $\left.a\right|_{r \tau} b$ if $b=a_{1} \cdots a_{i-1} \cdot a \cdot a_{i+1} \cdots a_{n}$ where $a_{1} \cdots a_{i-1} \cdot a \cdot a_{i+1} \cdots a_{n}$ is a $r \tau$-factorization. However, as we shall see, there are several problems with this definition. So we make the following two definitions.

Definition 3.5 Let $D$ be an integral domain and $\tau$ a relation on $D^{\sharp}$. We say that $a$ reduced $\tau$-divides $b$, denoted $\left.a\right|_{r} \tau$, if $b=a_{1} \cdots a_{i-1} \cdot a \cdot a_{i+1} \cdots a_{n}$ where $a_{1} \cdots a_{i-1} \cdot a \cdot a_{i+1} \cdots a_{n}$ is a $r \tau$-factorization of $b$. We say that $a$ weakly reduced $\tau$-divides $b$, denoted $\left.a\right|_{\text {wr }} \tau b$, if either (1) $\left.a\right|_{r} \tau b$ or (2) $a \sim b$.

Our next proposition concerns the relations $\left.\right|_{\tau},\left.\right|_{r} \tau$ and $\left.\right|_{w r} \tau$.

Proposition 3.6 Let $D$ be an integral domain and $\tau$ a symmetric relation on $D^{\sharp}$. Let $a, b \in D^{\sharp}$.

(1) $\left.\left.\left.a\right|_{r} \tau \Rightarrow a\right|_{w r} \tau \Rightarrow a\right|_{\tau} b$.

(2) The following are equivalent.

(a) $\left.\left.a\right|_{r} \tau b \Leftrightarrow a\right|_{\tau} b$ for all $a, b \in D^{\sharp}$,

(b) $\left.\left.a\right|_{r} \tau b \Leftrightarrow a\right|_{w r} \tau b$ for all $a, b \in D^{\sharp}$, and

(c) $D$ is a field or $U(D)=\{1\}$.

(3) Suppose that $\tau$ is associate preserving and that $\left.a\right|_{\tau} b$, so $b=\lambda a_{1} \cdots a_{n-1} a=\left(\lambda a_{1}\right) \cdot a_{2} \cdots a_{n-1} \cdot a, a$ ${ }_{r} \tau$-factorization. If $n>1,\left.a\right|_{r \tau} b$ and hence $\left.a\right|_{w r} \tau$. If $n=1, b=\lambda a$, so $\left.a\right|_{w r} \tau$, but $\left.a\right|_{r \tau} b \Leftrightarrow \lambda=1$.

(4) $\left.\left.a\right|_{\tau} b \Leftrightarrow a\right|_{w r}$ b for all $a, b \in D^{\sharp}$ if $\tau$ is associate preserving. If $\tau$ is multiplicative, the converse is true.

Proof (1) Clear. (2) (c) $\Rightarrow(a)$, (b) Clear. $(a) \Rightarrow(b),(c)$ Suppose that $D$ is not a field, so there is an $a \in D^{\sharp}$, and let $\lambda \in U(D)-\{1\}$. Thus $\left.a\right|_{\tau} \lambda a$ and $\left.a\right|_{w r} \lambda a$, but $a \nmid_{r} \tau \lambda a$. (3) Clear. (4) The first statement follows from (3). Suppose that $\tau$ is multiplicative but not associate preserving. So there exist $a, b \in D^{\sharp}$ and $\lambda \in U(D)$ with $a \tau b$ but $a / \tau(\lambda b)$. Now certainly $\left.a\right|_{\tau}(\lambda a b)$. But $a \nmid_{w r} \tau(\lambda a b)$. For $\left.a\right|_{w r} \tau(\lambda a b)$ implies $\left.a\right|_{r} \tau(\lambda a b)$ or $a \sim(\lambda a b)$. Now if $\left.a\right|_{r} \tau(\lambda a b)$, then $\lambda a b=a \cdot c$ where $a \tau c$ or $\lambda a b=a$. The first case $\lambda a b=a c$ gives $c=\lambda b$, so $a \tau \lambda b$, a contradiction. The second case, $\lambda a b=a$ gives that $b$ is a unit, also a contradiction. Likewise $a \sim \lambda a b$ leads to a contradiction.

If $\tau$ is not multiplicative the converse of the first statement of (4) does not hold in general. Define $\tau$ on $\mathbb{Z}^{\sharp}$ by $\pm 2 \tau \pm 2, \pm 2 \tau \pm 4, \pm 2 \tau 8, \pm 4 \tau 8$, and $b \tau a$ whenever $a \tau b$. Note that $\tau$ is neither associate preserving nor multiplicative, since $(2,-8),(2,16) \notin \tau$. However $\left.\left.a\right|_{\tau} b \Leftrightarrow a\right|_{w r} \tau$ for all $a, b \in \mathbb{Z}^{\sharp}$. 
Corollary 3.7 Let $D$ be an integral domain and $\tau$ a symmetric relation on $D^{\sharp}$.

(1) The ${ }_{r} \tau$-factorization theory on $D$ with $\left.\right|_{r} \tau$ coincides with the $\tau$-factorization theory on $D$ if and only if $D$ is a field or $U(D)=\{1\}$.

(2) If $D$ is associate preserving, then the $r \tau$-factorization theory on $D$ with $\left.\right|_{\text {wr }} \tau$ coincides with the $\tau$-factorization theory on D. If $\tau$ is multiplicative, the converse is true.

Recall that $a \in D^{\sharp}$ is a $\left.\right|_{\tau}$-prime if whenever $\left.a\right|_{\tau} \lambda a_{1} \cdots a_{n}, \lambda a_{1} \cdots a_{n}$ a $\tau$-factorization, then $\left.a\right|_{\tau} a_{i}$ for some $i$.

Definition 3.8 Let $D$ be an integral domain and $\tau$ a relation on $D^{\sharp}$. We define $a \in D^{\sharp}$ to be a (weakly) reduced $\tau$-divides prime, $\left.\right|_{r \tau}$-prime $\left(\left.\right|_{w r} \tau\right.$-prime $)$, if whenever $\left.a\right|_{r} \tau a_{1} \cdots a_{n}\left(\left.a\right|_{w r} \tau a_{1} \cdots a_{n}\right), a_{1} \cdots a_{n}$ a ${ }_{r} \tau$-factorization, then $\left.a\right|_{r} \tau a_{i}\left(\left.a\right|_{w r} \tau a_{i}\right)$ for some $i$.

It is easily checked that a $\left.\right|_{w r} \tau$-prime or $\left.\right|_{r} \tau$-prime is a ${ }_{r} \tau$-atom. In fact, a $\left.\right|_{w r} \tau$-prime is even a $\tau$-atom. However, a $\left.\right|_{\tau}$-prime need not be a $\left.\right|_{w r} \tau$-prime nor a $\left.\right|_{r} \tau$-prime. For with the relation $\tau_{3}$ on $\mathbb{Z}^{\sharp}$ defined by $a \tau_{3} b \Leftrightarrow a \equiv b$ mod 3 (see Example 2.3(4)), 5 is a $\left.\right|_{\tau_{3}}$-prime (Example 3.9), but $\left.5\right|_{w_{r} \tau_{3}} 5 \cdot\left(5 \cdot 7^{2}\right)=(5 \cdot 7) \cdot(5 \cdot 7)$, while $5 \nmid_{w r} \tau_{3} 5.7$ with similar relations holding for $\left.\right|_{r} \tau_{3}$. Also, 7 is a $\left.\right|_{w r} \tau_{3}$-prime, but not a $\left.\right|_{r} \tau_{3}$-prime and its associate -7 is not a $\left.\right|_{w r} \tau_{3}$-prime. A $\left.\right|_{r} \tau$-prime need not be a $\left.\right|_{w r} \tau$-prime. For example, define $\tau$ on $\mathbb{Z}^{\sharp}$ by $a \tau b \Leftrightarrow a, b \geq 2$. Now -4 is vacuously a $\left.\right|_{r \tau}$-prime, but -4 is not a $\left.\right|_{w r} \tau$-prime since $-\left.4\right|_{w r} \tau=2 \cdot 2$, but $-4 \nmid_{w r} \tau 2$. The same example also shows that a $\left.\right|_{r} \tau$-prime need not be a $\left.\right|_{\tau}$-prime nor a $\tau$-atom and that an associate of a $\left.\right|_{r \tau}$-prime need not be a $\left.\right|_{r \tau}$-prime. Finally, a $\left.\right|_{w r} \tau$-prime need not be a $\left.\right|_{\tau}$-prime. Define $\tau$ on $\mathbb{Z}^{\sharp}$ by $2 \tau 16,16 \tau 2,-4 \tau 8$ and $8 \tau-4$. Then 16 is a $\left.\right|_{w r} \tau$-prime but not a $\left.\right|_{\tau}$-prime as $\left.16\right|_{\tau} 32=(-1)(-4) 8$ but $16 \nmid_{\tau} 8,-4$. The following example illustrates the differences between $\tau$-factorizations and ${ }_{r} \tau$-factorizations.

Example 3.9 Let $\tau_{3}$ be the relation on $\mathbb{Z}^{\sharp}$ defined by $a \tau_{3} b \Leftrightarrow a \equiv b \bmod 3$. So $\tau_{3}$ is not associate preserving.

(1) $\tau_{3}$-factorizations

(a) The $\tau_{3}$-atoms are $\pm p, p$ a prime and $\pm 3 n, n \geq 2,3 \nmid n$.

(b) The $\tau_{3}$-primes are $\pm p, p$ a prime and $\pm 3 p, p$ a prime, $p \neq 3$.

(c) The $\left.\right|_{\tau_{3}}$-primes are $\pm p, p$ a prime, $p \neq 3$.

(d) $\mathbb{Z}$ is $\tau_{3}$-atomic, even a $\tau_{3}$-HFD, but not a $\tau_{3}$-UFD.

(2) ${ }_{r} \tau_{3}$-factorizations

(a) The ${ }_{r} \tau_{3}$-atoms are $\pm p, p$ a prime; $\pm 3 n, n \geq 2,3 \nmid n ; p q, p, q$ primes, $p \not \equiv q \bmod 3, p, q \neq$ $3 ;-p q, p, q$ primes, $p \equiv q \bmod 3, p, q \neq 3$.

(b) The ${ }_{r} \tau_{3}$-primes are $\pm p, p$ a prime and $\pm 3 p, p \neq 3$ a prime.

(c) The $\left.\right|_{r} \tau_{3}$-primes: none.

The $\left.\right|_{w r} \tau_{3}$-primes are $p$, a prime, $p \equiv 1 \bmod 3$ and $-p, p$ a prime, $p \equiv 2 \bmod 3$.

(d) $\mathbb{Z}$ is ${ }_{r} \tau_{3}$-atomic, but it is not a ${ }_{r} \tau_{3}$-HFD as $(7 \cdot 2) \cdot(7 \cdot 2) \cdot(7 \cdot 2)=(-49) \cdot 2 \cdot 2 \cdot 2 \cdot(-7)$ are $r \tau$-atomic factorizations of length 3 and 5 .

The fact that there are no $\left.\right|_{r} \tau_{3}$-primes is a special case of the following result.

Proposition 3.10 Let $D$ be an integral domain with $\operatorname{char}(D) \neq 2$ and let $\tau$ be a reflexive, symmetric relation on $D^{\sharp}$. Then $D$ has no $\left.\right|_{r} \tau$-primes. Thus $\mathbb{Z}$ has no $\left.\right|_{r} \tau_{n}$-primes for any $n \geq 0$.

Proof Let $a \in D^{\sharp}$. Note that $a \neq-a$ since $\operatorname{char}(D) \neq 2$. Now $\left.a\right|_{r} \tau a \cdot a=(-a) \cdot(-a)$, but $a \nmid_{r} \tau(-a)$. Hence $a$ cannot be a $\left.\right|_{r} \tau$-prime.

All of the material in Sect. 2 concerning $\tau$-factorization has an analog for ${ }_{r} \tau$-factorization (in fact, two analogs in some cases since we can use either $\left.\right|_{r} \tau$ or $\left.\right|_{w r} \tau$ ). We have seen (Corollary 3.7) that for $\tau$ associate preserving, we can omit the leading unit in a $\tau$-factorization (that is, use ${ }_{r} \tau$-factorization) once we replace $\left.\right|_{r} \tau$ by $\left.\right|_{w r}$. This, along with Proposition 3.10, suggests that when working with ${ }_{r} \tau$-factorizations we should replace $\left.\right|_{r} \tau$ by $\left.\right|_{w r} \tau$. But this in turn suggests that $\tau$-factorization is preferable over ${ }_{r} \tau$-factorization since we can use the natural relation $\left.\right|_{\tau}$. As illustrated in Example 3.9, the theory of $\tau$-factorization is often "cleaner" than the corresponding theory of $r$-factorization. For example, having an associate of a $\tau$-atom remain a $\tau$-atom is certainly a desirable property. Finally, the leading unit in a $\tau$-factorization allows the flexibility in constructing some natural factorizations as pointed out in Example 2.3(3). 


\section{4 Г-Factorizations}

In this section we give an introduction to $\Gamma$-factorizations. $\Gamma$-factorizations were first studied in [11] and this investigation is continued in [9]. Many results not stated here are given in [9] and [11] which reveal the power, generality, and usefulness of the $\Gamma$-factorization framework. Let $D$ be an integral domain with quotient field $K$. We maintain the notation from Sects. 2 and 3 with the exception that $\tau$ need not be symmetric. For $a \in D^{\sharp}$, we call $a=\lambda a_{1} \cdots a_{n}, n \geq 1, \lambda \in U(D), a_{i} \in D^{\sharp}$ a factorization of $a$ and a reduced factorization of a is $a=a_{1} \cdots a_{n}, n \geq 1, a_{i} \in D^{\sharp}$. Note that the order of the factors matters. In the case $n=1$ we call the (reduced) factorization $a=\lambda\left(\lambda^{-1} a\right)(a=a)$ a (reduced) trivial factorization. We will consider a reduced factorization to be a factorization where $\lambda=1$, and conversely. For $a \in D^{\sharp}$ let $\operatorname{fact}(a)(\operatorname{resp} ., \operatorname{rfact}(a))$ be the set of factorizations of $a$ (resp., reduced factorizations of $a$ ). And let $\operatorname{tfact}(a)(\operatorname{resp} ., \operatorname{trfact}(a)$ ) be the set of trivial factorizations of $a$ (resp., trivial reduced factorizations of $a$ ). Finally, fact $(D)=\cup_{a \in D^{\sharp}} \operatorname{fact}(a), \operatorname{rfact}(D)=$ $\cup_{a \in D^{\sharp}} \operatorname{rfact}(a)$, $\operatorname{tfact}(D)=\cup_{a \in D^{\sharp}} \operatorname{tfact}(a)$ and $\operatorname{trfact}(D)=\cup_{a \in D^{\sharp}} \operatorname{trfact}(a)$.

Remark 2 We could have more rigorously defined a factorization in $D$ to be the "formal word" $\left(\lambda, a_{1}, \ldots, a_{n}\right.$, $1,1, \ldots)$ where $\lambda \in U(D), n \geq 1$ and $a_{i} \in D^{\sharp}$. A reduced factorization in $D$ is a factorization $\left(1, a_{1}, \ldots, a_{n}, 1\right.$, $1, \ldots)$. Let fact $(D)$ be the set of these factorizations in $D$. Define $\pi: f$ act $(D) \longrightarrow D^{\sharp}$ by $\pi\left(\left(\lambda, a_{1}, \ldots, a_{n}, 1\right.\right.$, $1, \ldots))=\lambda a_{1} \cdots a_{n}$. If $\left(\lambda, a_{1}, \ldots, a_{n}, 1,1, \ldots\right) \in \operatorname{fact}(D)$ with $\pi\left(\left(\lambda, a_{1}, \ldots, a_{n}, 1,1, \ldots\right)\right)=a$ we call $\left(\lambda, a_{1}, \ldots, a_{n}, 1,1, \ldots\right)$ a factorization of $a$ and write $a=\lambda a_{1} \cdots a_{n}$.

Definition 4.1 Let $\Gamma \subseteq f a c t(D)$. An element of $\Gamma$ (resp., $\Gamma(a):=\Gamma \cap f a c t(a)$ ) is called a $\Gamma$-factorization (resp., $\Gamma$-factorization of $a$ ). Let $a, b \in D^{\sharp}$. We say that $a$ (weakly) $\Gamma$-divides $b$, written $\left.a\right|_{\Gamma} b\left(\left.a\right|_{w} \Gamma b\right)$, if there exists a $\Gamma$-factorization of $b, b=\lambda a_{1} \cdots a_{n}$, where some $a_{i}=a$ (or $a \sim b$ ). We also say that $a_{i}$ is a $\Gamma$-factor $o f b$. An element $a \in D^{\sharp}$ is $\Gamma$-irreducible or a $\Gamma$-atom if $\Gamma \cap \operatorname{fact}(a) \subseteq \operatorname{tfact}(a)$. We denote the set of $\Gamma$-atoms of $D$ by atom( $\Gamma)$. A $\Gamma$-atomic factorization of $a$ is a $\Gamma$-factorization $a=\lambda a_{1} \cdots a_{n}$ where each $a_{i}$ is a $\Gamma$-atom and $D$ is said to be $\Gamma$-atomic if each $a \in D^{\sharp}$ with $\Gamma(a) \neq \emptyset$ has a $\Gamma$-atomic factorization. An element $a \in D^{\sharp}$ is a $\Gamma$-prime (resp., $\left.\right|_{\Gamma}$-prime, $\left.\right|_{w} \Gamma$-prime) if whenever $a \mid \lambda a_{1} \cdots a_{n}$ (resp., $\left.a\right|_{\Gamma} \lambda a_{1} \cdots a_{n},\left.a\right|_{w} \Gamma \lambda a_{1} \cdots a_{n}$ ), a $\Gamma$-factorization, then $a \mid a_{i}$ (resp., $\left.a\right|_{\Gamma} a_{i},\left.a\right|_{w} \Gamma a_{i}$ ) for some $i$. A proper ideal $I$ of $D$ is called a $\Gamma$-prime ideal if whenever $\lambda a_{1} \cdots a_{n} \in I, \lambda a_{1} \cdots a_{n} \in \Gamma$, we have $a_{i} \in I$ for some $i$. We denote the set of $\Gamma$-prime ideals by $\operatorname{Spec}_{\Gamma}(D)$ and the set of $\Gamma$-prime (resp., $\left.\right|_{\Gamma}$-prime, $\left.\right|_{w} \Gamma$-prime) elements of $D$ by $\operatorname{pSpec}_{\Gamma}(D)$ (resp., $\left.\operatorname{pSpec}_{\mid \Gamma}(D), \operatorname{pSpec}_{\left.\right|_{w \Gamma}}(D)\right)$.

Note that according to our definition of a $\Gamma$-atomic domain $D$, an element of $D^{\sharp}$ need not to be a $\Gamma$-product of $\Gamma$-atoms. In fact, if $\Gamma=\emptyset$, every element of $D^{\sharp}$ is a $\Gamma$-atom, but no element of $D^{\sharp}$ has a $\Gamma$-atomic factorization. We will give some examples of $\Gamma$-factorizations later. For the moment we content ourselves with two examples. Let $\tau$ be a symmetric relation on $D^{\sharp}, D$ an integral domain. Let $\Gamma_{\tau}$ (resp., $\Gamma_{r} \tau$ ) be the set of all $\tau$-factorizations (resp., reduced $\tau$-factorizations) on $D$. So a $\Gamma_{\tau}$-factorization (resp., $\Gamma_{r} \tau$-factorization) is just a $\tau$-factorization (resp., reduced $\tau$-factorization). We have $\left.\left.a\right|_{\Gamma_{\tau}} b \Leftrightarrow a\right|_{\tau} b,\left.\left.a\right|_{\Gamma_{r \tau}} b \Leftrightarrow a\right|_{r} b$ and $\left.\left.a\right|_{w} \Gamma_{r \tau} b \Leftrightarrow a\right|_{w r} \tau$. Similar statements hold for $\tau$-prime, ${ }_{r} \tau$-prime, $\left.\right|_{\tau}$-prime, $\left.\right|_{r} \tau$-prime and $\left.\right|_{w r} \tau$-prime.

Observe that $a \in D^{\sharp}$ is a $\Gamma$-prime if and only if $(a)$ is a $\Gamma$-prime ideal. Thus an associate of a $\Gamma$-prime is again a $\Gamma$-prime. This is not true for $\Gamma$-atoms or $\left.\right|_{\Gamma}$-primes as seen by taking $\Gamma$ to be $\Gamma_{r} \tau$. The notions of $\Gamma$-atom, $\Gamma$-prime and $\left.\right|_{\Gamma}$-prime are all special cases of a $\Gamma_{1}-\Gamma_{2}-\Gamma_{3}$-prime.

Definition 4.2 Let $D$ be an integral domain. Let $\Gamma_{1}, \Gamma_{2}, \Gamma_{3} \subseteq$ fact $(D)$. We call $a \in D^{\sharp}$ a $\Gamma_{1}-\Gamma_{2}-\Gamma_{3}-$ prime if for each $\Gamma_{1}$-factorization $\lambda a_{1} \cdots a_{n},\left.a\right|_{\Gamma_{2}} \lambda a_{1} \cdots a_{n}$ implies $\left.a\right|_{\Gamma_{3}} a_{i}$ for some $i$. We denote the set of $\Gamma_{1}-\Gamma_{2}-\Gamma_{3}$-primes by $\operatorname{pSpec}_{\Gamma_{1}-\Gamma_{2}-\Gamma_{3}}(D)$.

Thus a $\Gamma$-fact $(D)$-fact $(D)$-prime is just a $\Gamma$-prime and a $\Gamma-\Gamma-\Gamma$-prime is a $\left.\right|_{\Gamma}$-prime. We next note that a $\Gamma$-atom is just a $\Gamma$-trfact $(D)$-tfact $(D)$-prime or $\Gamma$-trfact $(D)$-fact $(D)$-prime.

Proposition 4.3 Let $D$ be an integral domain and $\Gamma \subseteq \operatorname{fact}(D)$.

(0) For $a, b \in D^{\sharp},\left.\left.a\right|_{\Gamma} b \Rightarrow a\right|_{w} \Gamma \Rightarrow a \mid b$.

(1) $\operatorname{tfact}(D) \subseteq \Gamma \Leftrightarrow a, b \in D^{\sharp}$ with $a \sim b$ implies $\left.a\right|_{\Gamma} b$.

(1r) $\left.\operatorname{trfact}(D) \subseteq \Gamma \Leftrightarrow a\right|_{\Gamma}$ a for all $a \in D^{\sharp}$.

(2) $\Gamma \subseteq \operatorname{tfact}(D) \Leftrightarrow$ for $a, b \in D^{\sharp},\left.a\right|_{\Gamma} b$ implies $a \sim b$.

(2r) $\Gamma \subseteq \operatorname{trfact}(D) \Leftrightarrow$ for $a, b \in D^{\sharp},\left.a\right|_{\Gamma} b$ implies $a=b$.

(3) $\Gamma=\operatorname{tfact}(D) \Leftrightarrow$ for $a, b \in D^{\sharp},\left.a \sim b \Leftrightarrow a\right|_{\Gamma} b$. 
(3r) $\Gamma=\operatorname{trfact}(D) \Leftrightarrow$ for $a, b \in D^{\sharp}, a=\left.b \Leftrightarrow a\right|_{\Gamma} b$.

(4) Let $a \in D^{\sharp}$. The following are equivalent:

(a) a is $\Gamma$-irreducible,

(b) $a$ is $\Gamma$-trfact $(D)$ - $\Gamma_{3}$-prime for some $\Gamma_{3} \subseteq$ fact $(D)$,

(c) $a$ is $\Gamma$-trfact $(D)$-tfact $(D)$-prime, and

(d) $a$ is $\Gamma$-trfact $(D)-\Gamma_{3}$-prime for every $\Gamma_{3} \supseteq \operatorname{tfact}(D)$.

(5) The following are equivalent:

(a) $\Gamma \subseteq \operatorname{tfact}(D)$,

(b) every element of $D^{\sharp}$ is a $\Gamma$-atom, and

(c) every element of $D^{\sharp}$ is a $\Gamma$-prime.

Proof (0) Clear. (1) $\Rightarrow$ ) Suppose $a \sim b$; so $b=\lambda a$ for some $\lambda \in U(D)$. But $b=\lambda a$ is a trivial factorization of $b$ and hence a $\Gamma$-factorization of $b$. So $\left.a\right|_{\Gamma} b$. $(\Leftarrow)$ Let $b=\lambda a$ be a trivial factorization of $b$. We need that $\lambda a$ is in $\Gamma$. Now $a \sim b$, so $\left.a\right|_{\Gamma} b$. So $b=\mu a_{1} \cdots a_{n}$ is a $\Gamma$-factorization where say $a_{i}=a$. So $\lambda a=b=\mu a_{1} \cdots a_{i-1} \cdot a \cdot a_{i+1} \cdots a_{n}$. If $n>1$, cancelling $a$ gives $\lambda=\mu a_{1} \cdots a_{i-1} a_{i+1} \cdots a_{n}$, a contradiction. So $n=1$ and hence $\lambda=\mu$, so $b=\lambda a$ is in $\Gamma$. The proof of (1r) is similar.

$(2)(\Rightarrow)$ Suppose $\left.a\right|_{\Gamma} b$; so $b=\lambda a_{1} \cdots a_{n}$ a $\Gamma$-factorization. But $\Gamma \subseteq \operatorname{tfact}(D)$ gives $n=1$ so $b \sim a$. $(\Leftarrow)$ Let $b=\lambda a_{1} \cdots a_{n}$ be a $\Gamma$-factorization. Now $\left.a_{1}\right|_{\Gamma} b$, so $a_{1} \sim b$. Hence $a_{1}=\mu b$ where $\mu \in U(D)$. So $b=\lambda(\mu b) \cdot a_{2} \cdots a_{n}$. So $n>1$ forces $a_{2}, \ldots, a_{n}$ to be units, a contradiction. Hence $n=1$, so $b=\lambda a_{1} \in$ tfact $(D)$. The proof of $(2 \mathrm{r})$ is similar.

(3) This follows from (1) and (2). The proof of (3r) is similar.

(4) $(b) \Rightarrow(a)$ Suppose that $a$ is $\Gamma$ - $\operatorname{trfact}(D)-\Gamma_{3}$-prime. Let $a=\lambda a_{1} \cdots a_{n}$ be a $\Gamma$-factorization of $a$. Then $\left.a\right|_{\operatorname{trfact}(D)} \lambda a_{1} \cdots a_{n}$. So $\left.a\right|_{\Gamma_{3}} a_{i}$ for some $i$. Hence $a \mid a_{i}$, say $a_{i}=b a, b \in D^{*}$. So $a=\lambda a_{1} \cdots a_{n}=$ $\lambda a_{1} \cdots a_{i-1} \cdot(b a) \cdot a_{i+1} \cdots a_{n}$. Hence $n=1$; so $a$ is $\Gamma$-irreducible. $(a) \Rightarrow(d)$ Suppose that $a$ is $\Gamma$-irreducible. Suppose that $\left.a\right|_{\operatorname{trfact}(D)} \lambda a_{1} \cdots a_{n}$ where $\lambda a_{1} \cdots a_{n}$ is a $\Gamma$-factorization. By (3r) $\left.a\right|_{\operatorname{trfact}(D)} \lambda a_{1} \cdots a_{n}$ implies $a=\lambda a_{1} \cdots a_{n}$. Now $a \Gamma$-irreducible gives $n=1$, so $a=\lambda a_{1}$. Hence $a \sim a_{1}$, so $\left.a\right|_{\Gamma_{3}} a_{1}$. So $a$ is a $\Gamma$-trfact $(D)-\Gamma_{3}$-prime. $(d) \Rightarrow(c) \Rightarrow(b)$ Clear.

(5) This follows from the definitions.

Our next proposition gives some properties of $\cup \Gamma_{\alpha}, \cap \Gamma_{\alpha}$ and $\Gamma_{2} \backslash \Gamma_{1}$ for $\Gamma_{\alpha}, \Gamma_{1}$, $\Gamma_{2} \subseteq$ fact $(D)$. Its simple proof is left to the reader.

Proposition 4.4 Let $D$ be an integral domain, $\Gamma_{1}, \Gamma_{2}, \Gamma_{\alpha} \subseteq f a c t(D)$ and $a, b \in D^{\sharp}$.

(1) $\left(\underset{\alpha}{\cup} \Gamma_{\alpha}\right)(a)=\cup_{\alpha} \Gamma_{\alpha}(a)$.

(2) $\left(\cap \Gamma_{\alpha}\right)(a)=\cap_{\alpha} \Gamma_{\alpha}(a)$.

(3) If $\Gamma_{1} \subseteq \Gamma_{2}$, then $\left.\left.a\right|_{\Gamma_{1}} b \Rightarrow a\right|_{\Gamma_{2}} b$.

(4) $\left(\Gamma_{2} \backslash \Gamma_{1}\right)(a)=\left(\Gamma_{2}(a)\right) \backslash\left(\Gamma_{1}(a)\right)$.

(5) $\left.\left.a\right|_{\left(\cup \Gamma_{\alpha}\right)} b \Leftrightarrow a\right|_{\Gamma_{\alpha}} b$ for some $\alpha$.

(6) $\left.\left.a\right|_{\left(\cap \Gamma_{\alpha}\right)} ^{\alpha} b \Rightarrow a\right|_{\Gamma_{\alpha}} b$ for all $\alpha$.

(7) If $\left.a^{\alpha}\right|_{\Gamma_{2}} b$ and $a \nmid_{\Gamma_{1}} b$, then $\left.a\right|_{\left(\Gamma_{2} \backslash \Gamma_{1}\right)} b$.

Example 4.5 Let $D$ be an integral domain and $\left\{I_{\alpha}\right\}$ a family of ideals of $D$. Define $a \tau_{I_{\alpha}} b \Leftrightarrow a-b \in I_{\alpha}$ (Example 2.3(4)) and let $\Gamma_{\tau_{I_{\alpha}}}$ be the set of $\tau_{I_{\alpha}}$-factorizations. Then $\bigcap_{\alpha} \Gamma_{\tau_{I_{\alpha}}}=\Gamma_{\tau_{\alpha} I_{\alpha}}$ In particular, for $D=\mathbb{Z}$ and natural numbers $n_{1}, \ldots, n_{s}$, we have $\Gamma_{\tau_{n_{1}}} \cap \cdots \cap \Gamma_{\tau_{n_{s}}}=\Gamma_{\tau_{m}}$ (or $\tau_{n_{1}} \cap \cdots \cap \tau_{n_{s}}=\tau_{m}$ ) where $m$ is the LCM of $n_{1}, \ldots, n_{s}$.

Example 4.6 Let $\Gamma_{\tau_{n}}$ be the set of all $\tau_{n}$-factorizations (Example 2.3(4)). Note that $\left.2\right|_{\Gamma_{\tau_{3}}} 14$ and $\left.2\right|_{\Gamma_{\tau_{5}}} 14$, but $2 \nmid \Gamma_{\tau_{3}} \cap \Gamma_{\tau_{5}}$ 14, or equivalently $2 \nmid \Gamma_{\tau_{15}}$ 14, hence the converse of (6) fails in general. The converse of (7) need not to hold. For example, let $\Gamma_{1}=\{2 \cdot 2 \cdot 3\}$ and $\Gamma_{2}=\{4 \cdot 3\}$ and note that $\left.3\right|_{\Gamma_{1}} 12,\left.3\right|_{\Gamma_{2}} 12$ and $\left.3\right|_{\Gamma_{2} \backslash \Gamma_{1}} 12$.

We next show how the various forms of "prime" elements behave with respect to $\cup_{\alpha} \Gamma_{\alpha}$ and $\cap_{\alpha} \Gamma_{\alpha}$.

Theorem 4.7 Let $D$ be an integral domain and let $\Gamma_{1}, \Gamma_{1}{ }^{\prime}, \Gamma_{2}, \Gamma_{2}{ }^{\prime}, \Gamma_{3}, \Gamma_{3}{ }^{\prime}, \Gamma_{\alpha}$ be subsets of fact $(D)$. Then the following hold.

(1) If $\Gamma_{1} \subseteq \Gamma_{2}$, then $\operatorname{Spec}_{\Gamma_{2}}(D) \subseteq \operatorname{Spec}_{\Gamma_{1}}(D)$. 
(2) $\operatorname{Spec}_{\cup \Gamma_{\alpha}}(D)=\bigcap_{\alpha} \operatorname{Spec}_{\Gamma_{\alpha}}(D)$.

(3) If $\Gamma_{1} \subseteq \Gamma_{1}{ }^{\prime}$, then $p \operatorname{Spec}_{\Gamma_{1}{ }^{\prime}-\Gamma_{2}-\Gamma_{3}}(D) \subseteq p \operatorname{Spec}_{\Gamma_{1}-\Gamma_{2}-\Gamma_{3}}(D)$.

(4) $\operatorname{pSpec}_{\left(\cup_{\alpha}\right)-\Gamma_{2}-\Gamma_{3}}(D)=\bigcap_{\alpha} p \operatorname{Spec}_{\Gamma_{\alpha}-\Gamma_{2}-\Gamma_{3}}(D)$.

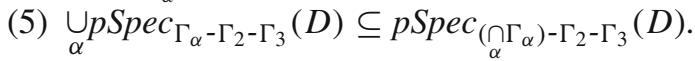

(6) If $\Gamma_{2} \subseteq \Gamma_{2}^{\prime}, p \operatorname{Spec}_{\Gamma_{1}-\Gamma_{2}{ }^{\prime}-\Gamma_{3}}(D) \subseteq p \operatorname{Spec}_{\Gamma_{1}-\Gamma_{2}-\Gamma_{3}}(D)$.

(7) $\operatorname{pSpec}_{\Gamma_{1}-\left(\cup_{\alpha} \Gamma_{\alpha}\right)-\Gamma_{3}}(D)=\bigcap_{\alpha} p \operatorname{Spec}_{\Gamma_{1}-\Gamma_{\alpha}-\Gamma_{3}}(D)$.

(8) $\cup_{\alpha} \operatorname{pSpec}_{\Gamma_{1}-\Gamma_{\alpha}-\Gamma_{3}}(D) \subseteq p \operatorname{Spec}_{\Gamma_{1}-\left(\cap \Gamma_{\alpha}\right)-\Gamma_{3}}(D)$.

(9) If $\Gamma_{3} \subseteq \Gamma_{3}{ }^{\prime}, p \operatorname{Spec}_{\Gamma_{1}-\Gamma_{2}-\Gamma_{3}}(D) \subseteq p \operatorname{Spec}_{\Gamma_{1}-\Gamma_{2}-\Gamma_{3}^{\prime}}(D)$.

(10) $\cup_{\alpha} \operatorname{Spec}_{\Gamma_{1}-\Gamma_{2}-\Gamma_{\alpha}}(D) \subseteq \operatorname{pSpec}_{\Gamma_{1}-\Gamma_{2}-\left(\cup \Gamma_{\alpha}\right)}(D)$.

(11) $\operatorname{pSpec}_{\Gamma_{1}-\Gamma_{2}-\left(\cap_{\alpha} \Gamma_{\alpha}\right)}(D) \subseteq \bigcap_{\alpha} p \operatorname{Spec}_{\Gamma_{1}-\Gamma_{2}-\Gamma_{\alpha}}(D)$.

(12) $\operatorname{pSpec}_{\Gamma_{2}}(D) \backslash p \operatorname{Spec}_{\Gamma_{1}}(D) \subseteq p \operatorname{Spec}_{\Gamma_{2} \backslash \Gamma_{1}}(D)$.

(13) $\operatorname{atom}\left(\cup_{\alpha} \Gamma_{\alpha}\right)=\underset{\alpha}{\operatorname{natom}}\left(\Gamma_{\alpha}\right)$.

(14) $\cup \operatorname{atom}\left(\Gamma_{\alpha}\right) \subseteq \operatorname{atom}\left(\cap \Gamma_{\alpha}\right)$.

(15) $\underset{\alpha}{\operatorname{atom}}\left(\Gamma_{2}\right) \backslash \operatorname{atom}\left(\Gamma_{1}\right) \stackrel{\alpha}{\subseteq} \operatorname{atom}\left(\Gamma_{2} \backslash \Gamma_{1}\right)$.

Proof (1) Let $I \in \operatorname{Spec}_{\Gamma_{2}}(D)$ and $\lambda a_{1} \cdots a_{n} \in I \cap \Gamma_{1} \subseteq I \cap \Gamma_{2}$, then $a_{i} \in I$ for some $i$. Hence $I \in \operatorname{Spec}_{\Gamma_{1}}(D)$.

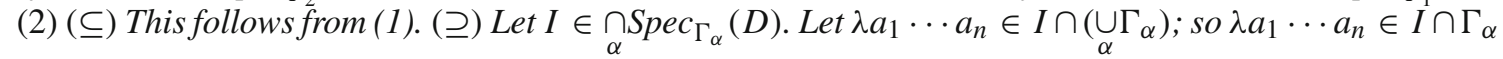

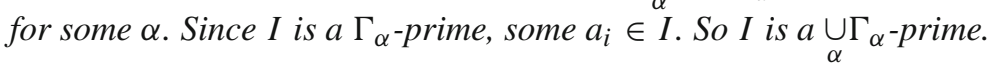

(3) The proof is similar to that of (1).

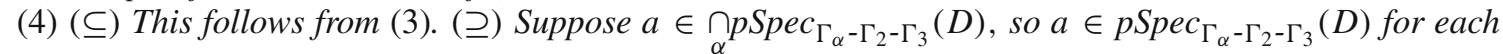
$\alpha$. Let $\left.a\right|_{\Gamma_{2}} \lambda a_{1} \cdots a_{n}, a \underset{\alpha}{\cup} \Gamma_{\alpha}$-factorization, that is, $\lambda a_{1} \cdots a_{n} \in \Gamma_{\alpha}$ for some $\alpha$. Hence a $\left.\right|_{\Gamma_{3}} a_{i}$ for some $i$.

(5) and (12) follow from (3). The proof of (6) and (9) are similar to that of (3).

(7) (ㄷ) Follows from (6). () Suppose $a \in \bigcap_{\alpha} p \operatorname{Spec}_{\Gamma_{1}-\Gamma_{\alpha}-\Gamma_{3}}(D)$, then $a \in p \operatorname{Spec}_{\Gamma_{1}-\Gamma_{\alpha}-\Gamma_{3}}(D)$ for each $\alpha$. Let a $\left.\right|_{\alpha} \Gamma_{\alpha} \lambda a_{1} \cdots a_{n}, a \Gamma_{1}$-factorization, which implies that a $\left.\right|_{\Gamma_{\alpha_{0}}} \lambda a_{1} \cdots a_{n}$ for some $\alpha_{0}$. Hence $\left.a\right|_{\Gamma_{3}} a_{i}$ for some $i$.

(8) Follows from (6).

(10) and (11) follow from (9).

(13), (14) and (15) follow from (3)-(5) and Proposition 4.3(4).

Example 4.8 Let $\Gamma_{1}=\{(-1) 2 \cdot 3\}$ and $\Gamma_{2}=\{-2 \cdot 3\}$, both subsets of fact $(\mathbb{Z})$. Then $\Gamma_{1} \cap \Gamma_{2}=\emptyset$, so atom $\left(\Gamma_{1} \cap \Gamma_{2}\right)=\mathbb{Z}^{\sharp}$. On the other hand, atom $\left(\Gamma_{1}\right) \cup$ atom $\left(\Gamma_{2}\right)=\mathbb{Z}^{\sharp}-\{-6\}$. So the reverse inclusion of (14) (and hence of (5)) does not hold in general. Now let $\Gamma_{1}=\Gamma_{2}$, then atom $\left(\Gamma_{2} \backslash \Gamma_{1}\right)=D^{\sharp}$, but atom $\left(\Gamma_{2}\right) \backslash$ atom $\left(\Gamma_{1}\right)=$ $\emptyset$. So the reverse inclusion of (15) [and hence of (12)] does not hold.

Proposition 4.9 Let $D$ be an integral domain. Let $\Gamma_{i}, \Gamma_{i}{ }^{\prime} \subseteq f a c t(D)$ for $i=1,2,3$ with $\Gamma_{1}{ }^{\prime} \subseteq \Gamma_{1}, \Gamma_{2}{ }^{\prime} \subseteq \Gamma_{2}$ and $\Gamma_{3}{ }^{\prime} \supseteq \Gamma_{3}$. Then a $\Gamma_{1}-\Gamma_{2}-\Gamma_{3}$-prime is a $\Gamma_{1}{ }^{\prime}-\Gamma_{2}{ }^{\prime}-\Gamma_{3}{ }^{\prime}-$ prime.

Proof Suppose a is $\Gamma_{1}-\Gamma_{2}-\Gamma_{3}$-prime. Suppose that a $\left.\right|_{\Gamma_{2}{ }^{\prime}} \lambda a_{1} \cdots a_{n} \in \Gamma_{1}{ }^{\prime}$ Now $\lambda a_{1} \cdots a_{n} \in \Gamma_{1}{ }^{\prime} \subseteq \Gamma_{1}$ and $\left.a\right|_{\Gamma_{2}} \lambda a_{1} \cdots a_{n}$ and $\left.\Gamma_{2}^{\prime} \subseteq \Gamma_{2} \Rightarrow a\right|_{\Gamma_{2}} \lambda a_{1} \cdots a_{n}$. So $a \Gamma_{1}-\Gamma_{2}-\Gamma_{3}-$ prime $\left.\Rightarrow a\right|_{\Gamma_{3}} a_{i}$ for some $i$. But then $\left.a\right|_{\Gamma_{3}^{\prime}} a_{i}$ since $\Gamma_{3}^{\prime} \supseteq \Gamma_{3}$.

Corollary 4.10 Let $D$ be an integral domain and let $\tau_{i}, \tau_{i}^{\prime}, i=1,2,3$, be symmetric relations on $D^{\sharp}$. Suppose that $\tau_{1} \geq \tau_{1}^{\prime}, \tau_{2} \geq \tau_{2}^{\prime}$ and $\tau_{3} \leq \tau_{3}^{\prime}$. If a is a $\tau_{1}-\tau_{2}-\tau_{3}$-prime, then a is a $\tau_{1}^{\prime}-\tau_{2}^{\prime}-\tau_{3}^{\prime}$-prime.

Corollary 4.11 Let $D$ be an integral domain and let $\Gamma_{1}, \Gamma_{2} \subseteq$ fact $(D)$ with $\Gamma_{1} \subseteq \Gamma_{2}$.

(1) $A \Gamma_{2}$-atom is a $\Gamma_{1}$-atom.

(2) $A \Gamma_{2}$-prime is a $\Gamma_{1}$-prime.

(3) A $\Gamma_{1}$-prime is a $\Gamma_{1}$-atom.

Proof (1), (2) Clear. (3) Let a be a $\Gamma_{1}$-prime. Now a is $\Gamma_{1}$-prime if and only if a is $\Gamma_{1}$-fact $(D)$-fact $(D)$-prime. So a is $\Gamma_{1}$-trfact $(D)$-fact $(D)$-prime, or equivalently, a is a $\Gamma_{1}$-atom, by Proposition 4.3.4. 
We can define "the" $\Gamma$-gcd $[a, b]_{\Gamma}$ for $a, b \in D^{\sharp}$. We say $[a, b]_{\Gamma}=d$ if (1) $\left.d\right|_{\Gamma} a$ and $\left.d\right|_{\Gamma} b$ and (2) if $\left.c\right|_{\Gamma} a$ and $\left.c\right|_{\Gamma} b$, then $\left.c\right|_{\Gamma} d$. If there does not exist $d \in D^{\sharp}$ with $\left.d\right|_{\Gamma} a$ and $\left.d\right|_{\Gamma} b$, we write $[a, b]_{\Gamma}=1$. This will be pursued in a future paper.

For $\tau$-factorization, we defined three additional conditions that the relation $\tau$ may satisfy: associate preserving, divisive, and multiplicative. These conditions on $\tau$ lead to desirable factorization properties. We next give several conditions that $\Gamma \subseteq \operatorname{fact}(D)$ may satisfy. These properties (along with others) were introduced in [11] and these properties (along with others) are further studied in [9].

Definition 4.12 Let $D$ be an integral domain and $\Gamma \subseteq$ fact $(D)$. Then we say $\Gamma$ is

(1) symmetric if for any $\lambda a_{1} \cdots a_{n} \in \Gamma$ and $\sigma \in S_{n}$, we have $\lambda a_{\sigma(1)} \cdots a_{\sigma(n)} \in \Gamma$,

(2) reflexive if for any $a \in D^{\sharp}$ and $n \geq 1$, we have the $n$-fold product $a \cdots a \in \Gamma$,

(3) associate preserving if for any $\lambda a_{1} \cdots a_{n} \in \Gamma$, then $\mu a_{1} \cdots\left(\mu_{i} a_{i}\right) \cdots a_{n}$ is also a $\Gamma$-factorization for any $\mu, \mu_{i} \in U(D)$,

(4) divisive if for any $\lambda a_{1} \cdots a_{n} \in \Gamma$ and $a \mid a_{i}\left(a \in D^{\sharp}\right)$ for $i \in\{1, \ldots, n\}$, we have $\lambda a_{1} \cdots a_{i-1} \cdot a$. $a_{i+1} \cdots a_{n} \in \Gamma$

(5) refinable if for any $\lambda a_{1} \cdots a_{n} \in \Gamma$ and $b_{i 1} \cdots b_{i m_{i}}$ a $\Gamma$-factorization of $a_{i}$, we have $\lambda b_{11} \cdots b_{1 m_{1}}$. $b_{21} \cdots b_{2 m_{2}} \cdots b_{n 1} \cdots b_{n m_{n}} \in \Gamma$

(6) combinable if for any $\lambda a_{1} \cdots a_{n} \in \Gamma$ and $k \in\{1,2, \ldots, n-1\}$, then $\lambda a_{1} \cdots a_{k-1} \cdot\left(a_{k} a_{k+1}\right) \cdot a_{k+2} \cdots a_{n} \in$ $\Gamma$,

(7) unital if whenever $\lambda a_{1} \cdots a_{n} \in \Gamma$, then $\mu a_{1} \cdots a_{n} \in \Gamma$ for any unit $\mu$,

(8) divisible if for any $\lambda a_{1} \cdots a_{n} \in \Gamma$, the subproduct $\lambda a_{i_{1}} a_{i_{2}} \cdots a_{i_{k}} \in \Gamma$ for each $1 \leq i_{1}<i_{2}<\cdots<i_{k-1}<$ $i_{k} \leq n$, and

(9) (reduced) normal if $(\operatorname{trfact}(D) \subseteq \Gamma) \operatorname{tfact}(D) \subseteq \Gamma$.

Let $\tau$ be a (symmetric) relation on $D^{\sharp}$. Then $\Gamma_{\tau}$ is symmetric, unital, divisible, reduced, and normal. If $\tau$ is reflexive (resp., associate preserving, divisive), then $\Gamma_{\tau}$ is reflexive (resp., associate preserving, divisive). If $\tau$ is divisive (resp., multiplicative), then $\Gamma_{\tau}$ is refinable (resp., combinable).

Suppose that $\Gamma$ is combinable. Then any $a \in D^{\sharp}$ with $\Gamma(a) \neq \emptyset$ which is not a $\Gamma$-atom has a $\Gamma$-factorization of length 2. If $\Gamma$ is divisive and combinable, then it is divisible; and if $\Gamma$ is divisive and unital, then it is associate preserving.

Proposition 4.13 Let $D$ be an integral domain and let $\Gamma$ be a unital subset of fact $(D)$. For $a \in D^{\sharp}$, the following are equivalent:

(1) a is a $\Gamma$-atom,

(2) $\lambda$ a is a $\Gamma$-atom for any $\lambda \in U(D)$,

(3) $a$ is a $\Gamma$-tfact $(D)$ - $\Gamma_{3}$-prime for some $\Gamma_{3} \subseteq$ fact $(D)$, and

(4) $\lambda$ a is a $\Gamma$-tfact $(D)$ - $\Gamma_{3}$-prime for some $\Gamma_{3} \subseteq$ fact $(D)$, for any $\lambda \in U(D)$.

Proposition 4.14 Let $D$ be an integral domain and $\Gamma \subseteq f a c t(D)$. Then the following statements hold.

(1) If $\Gamma$ is normal, then $\left.\mu a\right|_{\Gamma} \lambda$ a for any $\mu, \lambda \in U(D)$.

(2) If $\left.a\right|_{\Gamma} b$ and $\left.b\right|_{\Gamma} a$, then $a \sim b$.

(3) Let $\Gamma$ be unital. If $\lambda \in U(D),\left.\left.a\right|_{\Gamma} b \Leftrightarrow a\right|_{\Gamma} \lambda b$.

(4) Suppose $\Gamma$ is associate preserving. If $\lambda, \mu \in U(D)$, then $\left.\left.a\right|_{\Gamma} b \Leftrightarrow \mu a\right|_{\Gamma} \lambda b$.

(5) Let $\Gamma$ be refinable and associate preserving. If $\left.a\right|_{\Gamma} b$ and $\left.b\right|_{\Gamma} c$, then $\left.a\right|_{\Gamma} c$.

Proposition 4.15 Let $D$ be an integral domain and $\Gamma_{1}, \Gamma_{2}, \Gamma_{\alpha} \subseteq$ fact $(D)$. The following hold.

(1) If $\Gamma_{1} \subseteq \Gamma_{2}$ and $\Gamma_{1}$ is reflexive (resp., normal, reduced), then $\Gamma_{2}$ is reflexive (resp., normal, reduced).

(2) If each $\Gamma_{\alpha}$ is reflexive (resp., symmetric, associate preserving, combinable, divisive, divisible, normal, reduced, unital), so are $\cup \Gamma_{\alpha}$ and $\cap \Gamma_{\alpha}$.

(3) If each $\Gamma_{\alpha}$ is refinable, then $\cap_{\alpha} \Gamma_{\alpha}$ is refinable.

Proof (1) Follows from the definition. (2) If each $\Gamma_{\alpha}$ is reflexive, (1) gives $\cup_{\alpha} \Gamma_{\alpha}$ is reflexive. Now, for any $a \in D^{\sharp}$, the $n$-fold product $a \cdots a \in \Gamma_{\alpha}$. Hence $\bigcap_{\alpha} \Gamma_{\alpha}$ is reflexive. The argument for normal or reduced is similar. 
Suppose $\Gamma_{\alpha}$ is symmetric for each $\alpha$ and let $\lambda a_{1} \cdots a_{n} \in \bigcap_{\alpha} \Gamma_{\alpha}$ (resp., in $\underset{\alpha}{\cup} \Gamma_{\alpha}$ ), then $\lambda a_{1} \cdots a_{n} \in \Gamma_{\alpha}$ for all $\alpha$ (resp., for some $\alpha$ ). So $\lambda a_{\sigma(1)} \cdots a_{\sigma(n)} \in \Gamma_{\alpha}$ for all $\alpha$ (resp., for some $\alpha$ ) and $\lambda a_{\sigma(1)} \cdots a_{\sigma(n)} \in \bigcap_{\alpha} \Gamma_{\alpha}$ (resp., $\lambda a_{\sigma(1)} \cdots a_{\sigma(n)} \in \Gamma_{\alpha} \subseteq \cup_{\alpha} \Gamma_{\alpha}$ ). The arguments for the other properties are similar.

(3) Suppose $\Gamma_{\alpha}$ is refinable for all $\alpha$. Let $\lambda a_{1} \cdots a_{n} \in \underset{\alpha}{\cap} \Gamma_{\alpha}$ and $a_{i}=b_{i 1} \cdots b_{i m_{i}} \in \underset{\alpha}{\cap} \Gamma_{\alpha}$, then both $\cap \Gamma_{\alpha}$-factorizations are $\Gamma_{\alpha}$-factorizations for each $\alpha$. Hence $\lambda b_{11} \cdots b_{1 m_{1}} \cdot b_{21} \cdots b_{2 m_{2}} \cdots b_{n 1} \cdots b_{n m_{n}}$ is a $\stackrel{\alpha}{\Gamma}_{\alpha}$-factorization for each $\alpha$, that is, $\lambda b_{11} \cdots b_{1 m_{1}} \cdot b_{21} \cdots b_{2 m_{2}} \cdots b_{n 1} \cdots b_{n m_{n}} \in \underset{\alpha}{\cap} \Gamma_{\alpha}$.

The converse of (2) fails in general. For example consider $\Gamma_{1}=\{(-1)(-6), 2 \cdot 3,(-1) \cdot(-2) \cdot(-3),(-1)$. $2 \cdot 3\}$ and $\Gamma_{2}=\{(-6), 2 \cdot(-3),(-2) \cdot 3,(-1) \cdot 2 \cdot(-3),(-1) \cdot(-2) \cdot 3\}$. Now, $\Gamma_{1} \cap \Gamma_{2}=\emptyset$ and $\Gamma_{1} \cup \Gamma_{2}=$ $\{(-6),(-1)(-6), 2 \cdot 3,2 \cdot(-3),(-2) \cdot 3(-1) \cdot 2 \cdot 3,(-1) \cdot(-2) \cdot(-3),(-1) \cdot 2 \cdot(-3),(-1) \cdot(-2) \cdot 3\}$ are both associate preserving (resp., divisive, unital), but neither $\Gamma_{1}$ nor $\Gamma_{2}$ is associate preserving (resp., divisive, unital). We can construct similar examples in which the sets are pairwise disjoint by splitting the property among the sets so the union has the desired property. We next give some examples of $\Gamma$-factorizations.

Example 4.16 Let $D$ be an integral domain.

(1) Let $\tau$ be a symmetric relation on $D^{\sharp}$. $\Gamma_{\tau}=\left\{\lambda a_{1} \cdots a_{n} \mid a_{i} \tau a_{j}\right.$ for $\left.i \neq j\right\}$ is the set of $\tau$-factorizations and $\Gamma_{r \tau}=\left\{a_{1} \cdots a_{n} \mid a_{i} \tau a_{j}\right.$ for $\left.i \neq j\right\}$ is the set of reduced $\tau$-factorizations. So $\left.\right|_{\Gamma_{\tau}}=\left.\right|_{\tau}$ and $\left.\right|_{\Gamma_{r} \tau}=\left.\right|_{r}$.

(2) $\Gamma=$ fact $(D)$. We have the usual factorization, $\left.\right|_{\Gamma}=\mid, \Gamma$-atom $=$ atom, and $\left.\right|_{\Gamma}$-prime $=\Gamma$-prime $=$ principal prime. Note that $\Gamma=\Gamma_{\tau}$ where $\tau=D^{\sharp} \times D^{\sharp}$. Of course $\Gamma$ satisfies all of the conditions given in Definition 4.12.

(3) $\Gamma=\emptyset$. Here every element of $D^{\sharp}$ is a $\Gamma$-atom and a $\Gamma$-prime. We never have $\left.a\right|_{\Gamma} b$; so each element is a $\left.\right|_{\Gamma}$-prime. Also, $[a, b]_{\Gamma}=1$. Note that $\Gamma \neq \Gamma_{\tau}$ for any relation $\tau$. Here $\Gamma$ vacuously satisfies all of the condition given in Definition 4.12 except (2) and (9).

(4) $\Gamma=\operatorname{tfact}(D)$. Here every element of $D^{\sharp}$ is a $\Gamma$-atom and a $\Gamma$-prime. Also, $\left.a\right|_{\Gamma} b \Leftrightarrow a \sim b$. So each $a \in D^{\sharp}$ is also a $\left.\right|_{\Gamma}$-prime. We have $[a, b]_{\Gamma}=1 \Leftrightarrow a \nsucc b$. Note that $\Gamma=\Gamma_{\tau}$ where $\tau=\emptyset$. Here $\Gamma$ satisfies all the conditions given in Definition 4.12 except (2).

(5) Let $\tau$ be a not necessarily symmetric relation on $D^{\sharp}$. Then $\lambda a_{1} \cdots a_{n} \in$ fact $(D)$ is an ordered $\tau$-factorization if $a_{i} \tau a_{j}$ for $i<j$ and $\left.a\right|_{\tau} ^{\text {ord }} b$ if $b=\lambda a_{1} \cdots a_{n}$ is an ordered $\tau$-factorization where some $a_{i}=a$. Take $\Gamma_{\tau}^{\text {ord }}$ to be the set of all ordered $\tau$-factorizations. So $\left.\left.a\right|_{\tau} ^{\text {ord }} b \Leftrightarrow a\right|_{\Gamma_{\tau}} ^{\text {ord }} b$. In a similar manner we can define a reduced ordered $\tau$-factorization, $\left.\right|_{r \tau} ^{\text {ord }}$ and $\left.\right|_{w r} ^{\text {ord }}$. If we let $\Gamma_{r \tau}^{\text {ord }}$ be the set of reduced ordered $\tau$-factorizations, then $\left.\right|_{r \tau} ^{\text {ord }}=\left.\right|_{\Gamma_{r \tau}^{\text {ord }}}$ and $\left.\right|_{w r \tau} ^{\text {ord }}=\left.\right|_{w} \Gamma_{r \tau}^{\text {ord }}$. As a concrete example, define $\tau$ on $D[x]^{\sharp}$ by $f \tau g \Leftrightarrow \operatorname{deg}(f) \leq \operatorname{deg}(g)$. Then an ordered $\tau$-factorization is a factorization $\lambda f_{1} \cdots f_{n}$ where $\lambda$ is a unit and $f_{i} \in D[x]^{\sharp}$ with $\operatorname{deg}\left(f_{1}\right) \leq \operatorname{deg}\left(f_{2}\right) \leq \cdots \leq \operatorname{deg}\left(f_{n}\right)$. Note that $\Gamma_{\tau}$ is not symmetric, divisive, refinable, nor combinable, but satisfies the other conditions given in Definition 4.12. As a second example we could consider factorizations in $\mathbb{Z}$ of the form $( \pm 1) 2^{n_{1}} \cdot 3^{n_{2}} \cdots p_{k}^{n_{k}}$ where $p_{i}$ is the $i$ th prime.

(6) Let $S$ be a non-empty subset of $D^{\sharp}$ and take $\tau=S \times S$, so $a \tau b \Leftrightarrow a, b \in S$ (Example 2.3(3)). Take $\Gamma_{S}=\left\{\lambda a_{1} \cdots a_{n} \mid a_{i} \in S, n \geq 1\right\}$. Note that for any unit $\lambda$ and $a \in D^{\sharp}, \lambda a \in \Gamma_{\tau}$, but $\lambda a \in \Gamma_{S} \Leftrightarrow a \in S$. In fact, $\Gamma_{\tau}=\Gamma_{S} \cup \operatorname{tfact}(D)$. Suppose $a \sim b$. Then $\left.a\right|_{\tau} b$, but $\left.a\right|_{\Gamma_{S}} \Leftrightarrow a \in S$. Here we have $\left.c\right|_{\tau} d \Leftrightarrow$ $\left.c\right|_{w} \Gamma_{S} d$. Note that $\operatorname{Spec}_{\Gamma_{\tau}}(D)=\operatorname{Spec}_{\Gamma_{S}}(D)$, atom $\left(\Gamma_{\tau}\right)=\operatorname{atom}\left(\Gamma_{S}\right), \operatorname{pSpec}_{\Gamma_{\tau}}(D)=\operatorname{pSpec}_{\Gamma_{S}}(D)$, and $\operatorname{pSpec}_{\mid \Gamma_{\tau}}(D)=\operatorname{pSpec}_{\left.\right|_{w} \Gamma_{S}}(D)$.

We next define the $\Gamma$ analogs of $\tau$-UFD, $\tau$-HFD, $\tau$-BFD, $\tau$-FFD and $\tau$-ACCP. Note that these definitions agree with the $\tau$-factorization definitions in the case where $\Gamma=\Gamma_{\tau}$.

Definition 4.17 An integral domain $D$ is called a $\Gamma$-unique factorization domain $(\Gamma-U F D)$ if $D$ is $\Gamma$-atomic and whenever $\lambda a_{1} \cdots a_{n}=\mu b_{1} \cdots b_{m}$ are two $\Gamma$-factorizations into $\Gamma$-atoms, then $n=m$ and after reordering (if necessary) $a_{i} \sim b_{i}$. We say $D$ is a $\Gamma$-half-factorial domain ( $\Gamma$-HFD) if $D$ is $\Gamma$-atomic and for any two $\Gamma$-atomic factorizations $\lambda a_{1} \cdots a_{n}=\mu b_{1} \cdots b_{m}$ we have $m=n$. The domain $D$ is called a $\Gamma$-bounded factorization domain $(\Gamma-B F D)$ if $D$ is $\Gamma$-atomic and for each $a \in D^{\sharp}$ there is a positive integer $N_{\Gamma}(a)$ so that for any $\Gamma$-factorization $\lambda a_{1} \cdots a_{n}$ of $a, n \leq N_{\Gamma}(a)$. We say that $D$ is a $\Gamma$-finite factorization domain $(\Gamma-F F D)$ if $D$ is $\Gamma$-atomic and each $a \in D^{\sharp}$ has only finitely many $\Gamma$-factorizations (up to order and associates) into $\Gamma$-atoms. We say that $D$ satisfies the $\Gamma$-ascending chain condition on principal ideals $(\Gamma-A C C P)$ if for any infinite sequence $\left\{a_{i}\right\}_{i=1}^{\infty}$ such that $\left.a_{i+1}\right|_{\Gamma} a_{i}$ there is a positive integer $N$ (depending on the sequence) with $a_{k+1} \sim a_{k}$ for each $k>N$. 
Note that $\Gamma$-UFD $\Rightarrow \Gamma$-HFD and $\Gamma$-FFD, and that $\Gamma$-HFD or $\Gamma$-FFD $\Rightarrow \Gamma$-BFD. If $\Gamma$ is divisive, unital and refinable, we have $\Gamma$-BFD $\Rightarrow \Gamma$-ACCP $\Rightarrow \Gamma$-atomic (see Theorem 4.19). We next give a $\Gamma$ analog of Proposition 2.8 that will be used in proving Theorem 4.19(4).

Theorem 4.18 Let $D$ be an integral domain and $\Gamma \subseteq f a c t(D)$ be divisible, divisive and unital. Suppose $p_{1} \cdots p_{n}=q_{1} \cdots q_{m}$ are two $\Gamma$-factorizations so that each $p_{i}$ is a $\Gamma$-atom and each $q_{i}$ is a $\Gamma$-prime (resp. $\left.\right|_{\Gamma}$-prime). Then $n=m$ and after reordering (if necessary) $p_{i} \sim q_{i}$. Moreover, if each $a \in D^{\sharp}$ has $a \Gamma$-factor-

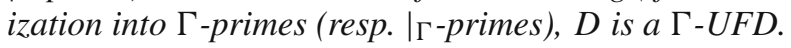

Proof First, let us assume that each $q_{i}$ is a $\left.\right|_{\Gamma}$-prime. Without loss of generality, say $\left.q_{m}\right|_{\Gamma} p_{n}$. So $p_{n}=$ $\alpha a_{1} \cdots q_{m} \cdots a_{k} \in \Gamma$. Since $p_{n}$ is a $\Gamma$-atom, $k=0$ and $p_{n}=\alpha q_{m}$. Cancelling gives two $\Gamma$-factorizations $\lambda q_{1} \cdots q_{m-1}=(\mu \cdot \alpha) p_{1} \cdots p_{n-1}$ (here we use divisible and unital). By induction, $m-1=n-1$ and after reordering (if necessary) $q_{i} \sim p_{i}$ for each $1 \leq i<n$. Therefore, $n=m$ and after reordering (if necessary) each $p_{i} \sim q_{i}$.

Now suppose that each $q_{i}$ is a $\Gamma$-prime. Then $q_{1} \mid p_{i}$ for some $i$, say $p_{i}=a_{i} q_{1}$. So $p_{1} \cdots p_{i-1} \cdot\left(a_{i} q_{1}\right)$. $p_{i+1} \cdots p_{n}=q_{1} \cdots q_{m}$. Cancelling $q_{1}$ gives $p_{1} \cdots p_{i-1} \cdot a_{i} \cdot p_{i+1} \cdots p_{n}=q_{2} \cdots q_{m}$ where both sides are again $\Gamma$-factorizations since $\Gamma$ is divisive and divisible. Now either $q_{2} \mid p_{j}$ for some $j \neq i$ or $q_{2} \mid a_{i}$. In the first case $p_{j}=a_{j} q_{2}$ and as before $p_{1} \cdots a_{i} \cdots a_{j} \cdots p_{n}=q_{3} \cdots q_{m}$ where both are $\Gamma$-factorizations. If $q_{2} \mid a_{i}$, then $a_{i}=a_{i}{ }^{\prime} q_{2}$ for some $a_{i}{ }^{\prime}$. So $p_{i}=a_{i}{ }^{\prime} q_{1} q_{2}$. In this case $p_{1} \cdots p_{i-1} \cdot a_{i}{ }^{\prime} \cdot p_{i+1} \cdots p_{n}=q_{3} \cdots q_{m}$ where both are again $\Gamma$ factorizations. Continuing in this manner, we can partition $q_{1}, q_{2}, \ldots, q_{m}$ (as an ordered set) into $\left\{q_{i j}\right\}$ so that $p_{i}=a_{i} q_{i 1} \cdots q_{i k_{i}}$ (need not be a $\Gamma$-factorization). Hence $\prod q_{i j}=q_{1} \cdots q_{m}$. Thus $q_{1} \cdots q_{m}=p_{1} \cdots p_{n}=\left(a_{1} q_{11} \cdots q_{1 k_{1}}\right) \cdots\left(a_{n} q_{n 1} \cdots q_{n k_{n}}\right)=a_{1} \cdots a_{n} q_{1} \cdots q_{m}$. Hence each $a_{i}$ is a unit. Since $\Gamma$ is divisive and unital $p_{i}=a_{i} q_{i 1} \cdots q_{i k_{i}}$ is a $\Gamma$-factorization of the $\Gamma$-atom $p_{i}$. Hence $k_{i}=1$. Therefore $n=m$ and after reordering (if necessary) $p_{i} \sim q_{i}$.

We end with the following theorem. Note that Theorem 4.19(4) gives a proof for Theorem 2.12 (which is much simpler than the proof given in [3]).

Theorem 4.19 Let $D$ be an integral domain and $\Gamma \subseteq f a c t(D)$.

(1) If D has ACCP, then D has $\Gamma$-ACCP.

(2) Suppose $\Gamma$ is divisive, unital and refinable. If $D$ satisfies $\Gamma$ - $A C C P$, then $D$ is $\Gamma$-atomic.

(3) Suppose $\Gamma$ is divisive, unital and refinable. If $D$ is a $\Gamma-B F D$, then $D$ satisfies $\Gamma-A C C P$.

(4) Suppose $\Gamma$ is divisive, divisible, unital and refinable. If $D$ is a UFD, then $D$ is a $\Gamma-U F D$.

Proof (1) If there is an infinite sequence $\left\{a_{i}\right\}_{i=1}^{\infty}$ with $\left.a_{i+1}\right|_{\Gamma} a_{i}$ that does not stabilize, then the same sequence contradicts the fact that $D$ has ACCP. Hence, there is a positive integer $N$ such that for each $k \geq N, a_{k} \sim a_{k+1}$ and $D$ has $\Gamma$-ACCP. (2) Let $a \in D^{\sharp}$, and suppose that it is not a $\Gamma$-atom and has no $\Gamma$-atomic factorization. So there is a $\Gamma$-factorization, say $a=b_{1} \cdots b_{n}$, which is not a $\Gamma$-atomic factorization. So some $b_{i}$, say $b_{1}$, is neither a $\Gamma$-atom nor has a $\Gamma$-atomic factorization. Say that $b_{1}=b_{11} \cdots b_{1 m_{1}}$ is neither a $\Gamma$ atom nor a $\Gamma$-atomic factorization of $b_{1}$. So $a=b_{11} \cdots b_{1 m_{1}} b_{2} \cdots b_{n}$ is not a $\Gamma$-atomic factorization of $a$. Again, we may suppose that $b_{11}=b_{21} \cdots b_{2 m_{2}}$ is neither a $\Gamma$-atom nor a $\Gamma$-atomic factorization of $b_{11}$. So $a=b_{21} \cdots b_{2 m_{2}} \cdot b_{12} \cdots b_{1 m_{1}} b_{2} \cdots b_{n}$ is not a $\Gamma$-atomic factorization of $a$. Continuing in this fashion, and we obtain the infinite sequence $\left\{b_{1}, b_{11}, b_{21}, b_{31}, \ldots\right\}$ with $\left.b_{11}\right|_{\Gamma} b_{1}$ and $\left.b_{(i+1) 1}\right|_{\Gamma} b_{i 1}$ that does not stabilize. This contradiction shows that every element is either a $\Gamma$-atom or has a $\Gamma$-atomic factorization. Therefore $D$ is $\Gamma$-atomic. (3) Let $D$ be a $\Gamma$-BFD. Suppose that there is an infinite sequence $\left\{a_{i}\right\}_{i=1}^{\infty}$ with $\left.a_{i+1}\right|_{\Gamma} a_{i}$ that does not stabilize. Now $\left.a_{2}\right|_{\Gamma} a_{1}$, so $a_{1}=\lambda b_{1} \cdots b_{i-1} \cdot a_{2} \cdot b_{i+1} \cdots b_{n} \in \Gamma$ where $n>1$ since $a_{1} \nsucc a_{2}$. Since $\left.a_{3}\right|_{\Gamma} a_{2}$, we have $a_{2}=\mu c_{1} \cdots c_{j-1} \cdot a_{3} \cdot c_{j+1} \cdots c_{m} \in \Gamma$ where $m>1$ since $a_{2} \nsucc a_{3}$. Since $\Gamma$ is divisive and unital, $\left(\mu c_{1}\right) \cdot c_{2} \cdots c_{j-1} \cdot a_{3} \cdot c_{j+1} \cdots c_{m} \in \Gamma$. Thus $\Gamma$ refinable gives $a_{1}=\lambda b_{1} \cdots b_{i-1} \cdot\left(\mu c_{1}\right) \cdot c_{2} \cdots c_{j-1} \cdot a_{3} \cdot c_{j+1} \cdots c_{m} \cdot b_{i+1} \cdots b_{n} \in \Gamma$. Continuing this process, we get arbitrarily long $\Gamma$-factorizations for $a_{1}$. This contradiction gives that $D$ satisfies $\Gamma$-ACCP. (4) Since a UFD has ACCP, $D$ is $\Gamma$-atomic by (2). Now, we show that each $\Gamma$-atom is a $\Gamma$-prime and by Theorem $4.18 D$ will be a $\Gamma$-UFD. Suppose that $a \in D^{\sharp}$ is a $\Gamma$-atom and $a \mid b_{1} \cdots b_{n} \in \Gamma$. Since $D$ is a UFD, it is a GCD domain and so $a=a_{1} \cdots a_{n}$, where each $a_{i} \in D^{*}$ and $a_{i} \mid b_{i}$. Now collect all those $a_{i}$ s that are non-units. Say $a_{i 1}, a_{i 2}, \ldots, a_{i k}$ are non-units and say that the product of the rest of them is $\alpha$. Then $a=\alpha a_{i 1} a_{i 2} \cdots a_{i k}$ is a $\Gamma$-factorization of $a$, which implies that $k=1$. Hence $a \mid b_{i 1}$ and $a$ is a $\Gamma$-prime.

Open Access This article is distributed under the terms of the Creative Commons Attribution License which permits any use, distribution, and reproduction in any medium, provided the original author(s) and the source are credited. 


\section{References}

1. Anderson, D.D.: Non-atomic factorization in integral domains. In: Chapman, S. (ed.): Arithmetical Properties of Commutative Rings and Monoids. Lecture Notes in Pure and Applied Math., vol. 241, pp. 1-21. CRC Press, Boca Raton (2005)

2. Anderson, D.D.; Anderson, D.F.; Zafrullah, M.: Factorization in integral domains. J. Pure Appl. Algebra 69, 1-19 (1990)

3. Anderson, D.D.; Frazier, A.: On a general theory of factorization in integral domains. Rocky Mt. J. Math. 41, 663-705 (2011)

4. Frazier, A.M.: Generalized factorizations in integral domains, PhD Thesis, The University of Iowa (2006)

5. Geroldinger, A.; Halter-Koch, F.: Non-unique Factorizations: Algebraic, Combinatorial and Analytic Theory. Pure and Applied Mathematics, vol. 278. Taylor and Francis, CRC Press, Boca Raton (2006)

6. Gilmer, R.: Multiplicative Ideal Theory. Queen's Papers in Pure and Appl. Math., vol. 90, Queen's University, Kingston, Ontario (1992)

7. Hamon, S.M.: Some topics in $\tau$-factorizations, PhD Thesis, The University of Iowa (2007)

8. Juett, J.: Two counterexamples in abstract factorization. Rocky Mt. J. Math. (in press)

9. Juett, J.: Some topics in $\Gamma$-factorizations in integral domains, PhD Thesis, The University of Iowa (in preparation)

10. McAdam, S.; Swan, R.G.: Unique comaximal factorization. J. Algebra 276, 180-192 (2004)

11. Ortiz-Albino, R.M.: On generalized nonatomic factorizations, PhD Thesis, The University of Iowa (2008)

12. Reinkoester, J.: Relative primeness, PhD Thesis, The University of Iowa (2010) 